\title{
Acid Ceramidase Deficiency in Mice Results in a Broad Range of Central Nervous System Abnormalities
}

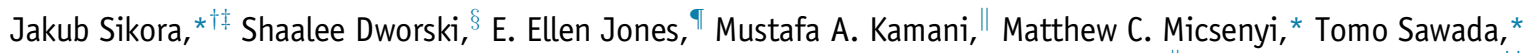
Pauline Le Faouder, ${ }^{* *}$ Justine Bertrand-Michel, ${ }^{* *}$ Aude Dupuy, ${ }^{* *}$ Christopher K. Dunn, Ingrid Cong Yang Xuan, ${ }^{\dagger \dagger}$ Josefina Casas,

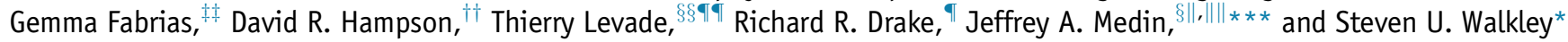

From the Dominick P. Purpura Department of Neuroscience,* Rose F. Kennedy Intellectual and Developmental Disabilities Research Center, Albert Einstein College of Medicine, Bronx, New York; the Institutes of Inherited Metabolic Disorders ${ }^{\dagger}$ and Pathology, ${ }^{\ddagger}$ Charles University, 1 st Faculty of Medicine, Prague, Czech Republic; the Institute of Medical Science ${ }^{\S}$ and the Departments of Pharmaceutical Sciences ${ }^{\dagger \dagger}$ and Medical Biophysics, $"\|\|$ University of Toronto,

Toronto, Ontario, Canada; the Department of Cell and Molecular Pharmacology, ${ }^{\top}$ Medical University of South Carolina, Charleston, South Carolina; the University Health Network," Toronto, Ontario, Canada; the MetaToul-Lipidomic Facility-MetaboHUB, ** INSERM UMR1048, Institute of Cardiovascular and Metabolic Diseases, Université Paul Sabatier-Toulouse III, Toulouse, France; the Research Unit on Bioactive Molecules, ${ }^{\ddagger \ddagger}$ Department of Biomedicinal Chemistry, Institute for Advanced Chemistry of Catalonia, Spanish National Research Council, Barcelona, Spain; the INSERM UMR1037, ${ }^{\S \S}$ Cancer Research Center of Toulouse, Universite Toulouse III Paul-Sabatier, Toulouse, France; the Metabolic Biochemistry Laboratory, " ${ }^{\top \uparrow}$ Federative Institute of Biology, Centre Hospitalier Universitaire de Toulouse, Toulouse, France; and the Medical College of Wisconsin,*** Milwaukee, Wisconsin

Accepted for publication December 8, 2016.

Address correspondence to Jeffrey A. Medin, Ph.D., Medical College of Wisconsin, 8701 Watertown Plank Rd., MFRC 3018, Milwaukee, WI 53226; or Steven U. Walkley, D.V.M., Ph.D., 1410 Pelham Parkway S., Room 618, Bronx, NY 10461. E-mail: jmedin@mcw. edu or steve.walkley@einstein. yu.edu.

\begin{abstract}
Farber disease is a rare autosomal recessive disorder caused by acid ceramidase deficiency that usually presents as early-onset progressive visceral and neurologic disease. To understand the neurologic abnormality, we investigated behavioral, biochemical, and cellular abnormalities in the central nervous system of $A s a h 1^{\mathrm{P} 361 \mathrm{R} / \mathrm{P} 361 \mathrm{R}}$ mice, which serve as a model of Farber disease. Behaviorally, the mutant mice had reduced voluntary locomotion and exploration, increased thigmotaxis, abnormal spectra of basic behavioral activities, impaired muscle grip strength, and defects in motor coordination. A few mutant mice developed hydrocephalus. Mass spectrometry revealed elevations of ceramides, hydroxy-ceramides, dihydroceramides, sphingosine, dihexosylceramides, and monosialodihexosylganglioside in the brain. The highest accumulation was in hydroxy-ceramides. Storage compound distribution was analyzed by mass spectrometry imaging and morphologic analyses and revealed involvement of a wide range of central nervous system cell types (eg, neurons, endothelial cells, and choroid plexus cells), most notably microglia and/or macrophages. Coalescing and mostly perivascular granuloma-like accumulations of storage-laden $\mathrm{CD} 68^{+}$microglia and/or macrophages were seen as early as 3 weeks of age and located preferentially in white matter, periventricular zones, and meninges. Neurodegeneration was also evident in specific cerebral areas in late disease. Overall, our central nervous system studies in $A s a h 1^{\text {P361R/P361R }}$ mice substantially extend the understanding of human Farber disease and suggest that this model can be used to advance therapeutic approaches for this currently untreatable disorder. (Am J Pathol 2017, 187: 864-883; http://dx.doi.org/10.1016/j.ajpath.2016.12.005)
\end{abstract}

Supported by National Institute of Neurological Diseases and Stroke Award 1F05 NS074790, Charles University Research Development Schemes (PRVOUK) grant P24/LF1/3, and OPPK grant CZ.2.16/3.1.00/ 24509 (J.S.); the Canadian Institutes of Health Research Biological Therapeutics Traineeship (S.D.); NIH grants 1R21NS078191-01A1 (J.A.M.), R01 HD045561 (S.U.W.), and P30 HD071593 (S.U.W.); Spanish Ministries of Science and Technology grant SAF2011-22444 and Economy and Competitiveness grant CTQ2014-54743-R (J.C. and G.F.); INSERM
(T.L.); Vaincre les Maladies Lysosomales (T.L. and J.A.M.); and the South Carolina SmartState Endowed Research program, National Cancer Institute grant P30 CA138313 and South Carolina Clinical and Translational Research Institute grants UL1 RR029882 and UL1 TR000062 (R.R.D.).

J.S., S.D., J.A.M., and S.U.W. contributed equally to this work.

Disclosures: None declared.

Current address of J.A.M., Medical College of Wisconsin, Milwaukee, WI. 
Farber disease (FD) (Online Mendelian Inheritance in Man no. 228000) is a rare autosomal recessive lysosomal disorder caused by mutations in the $A S A H 1$ gene ( $8 p 22)$ that encodes acid ceramidase (ACDase). ACDase is a key lysosomal enzyme of sphingolipid catabolism that converts ceramide (Cer) into sphingosine (Sph) and a free fatty acid. The FD clinical phenotype is variable, with the most common variant manifesting with progressive neurovisceral disease that is associated with early-onset subcutaneous nodules, progressive arthritis with joint deformities, and laryngeal hoarseness. ${ }^{1}$ At the tissue level, FD is characterized by accumulations of storage-laden macrophages. Other nonneuronal cells, such as hepatocytes, endothelial cells, sweat gland epithelial cells, fibroblasts, and/or Schwann cells, ${ }^{2-4}$ also have subcellular storage pathologic features. Although variable among the different cell types, ultrastructural storage profiles are characteristic, including comma-shaped curvilinear tubular profiles [curvilinear tubular bodies (CTBs) or Farber bodies]; empty banana, spindle, or needle-like bodies; and zebra-like bodies. ${ }^{4}$ Even though the most severely affected FD patients present with substantial neurologic deficits, an understanding of central nervous system (CNS) disease is limited. ${ }^{1,5,6}$ Importantly, an additional clinical variant in which a neuronal phenotype is not associated with classic FD symptoms has been recently described by Zhou et al. ${ }^{7}$ That study identified six patients with spinal muscular atrophy and progressive myoclonic epilepsy, with each individual carrying ASAH1 mutations and having reduced ACDase activity. Since that initial description, an additional five FD patients with spinal muscular atrophy and progressive myoclonic epilepsy have been described. ${ }^{8-11}$ Those studies suggest that FD may be more common than previously believed and that, rather than occurring as discrete variants or subtypes, FD actually occurs as a broad, continuous spectrum of clinical phenotypes.

To study the broad effect of ACDase deficiency in the mammalian model system and the mechanisms of FD progression, we recently generated an Asahl knock-in mouse model that carries a P361R ACDase mutation corresponding to the $\mathrm{P} 362 \mathrm{R}$ patient variant previously identified by Li et al. ${ }^{12}$ Although heterozygous (Het) mice appear unaffected and similar in all measures to wild-type (WT) mice, mice homozygous for the P361R mutation $\left(\right.$ Asahl $^{\mathrm{P} 361 \mathrm{R} / \mathrm{P} 361 \mathrm{R}}$, Hom Farber) develop a progressive and lethal phenotype (survival is 7 to 13 weeks) with recapitulation of major aspects (eg, reduced ACDase activity and bulk Cer accumulation in tissues) of human FD. ${ }^{13}$ Important for the relevance of the model to human disease, Hom Farber mice replicate the macrophage aspect of tissue pathologic findings observed in FD patients. Furthermore, a follow-up study identified substantial dysregulation of the hematopoietic microenvironment in the Hom Farber mice. ${ }^{14}$ Given the critical need to explore and understand the mechanisms underlying the neurologic defects observed among FD patients, in this current study we describe the hallmark behavioral, biochemical, and tissue and cell pathologic abnormalities in the CNS of Asah1 ${ }^{\mathrm{P} 361 \mathrm{R} / \mathrm{P} 361 \mathrm{R}}$ mice.

\section{Materials and Methods}

\section{Animals}

Mice deficient in ACDase activity (Asah1 $\left.{ }^{\mathrm{P} 361 \mathrm{R} / \mathrm{P} 361 \mathrm{R}}\right)$ were generated by our group. ${ }^{13}$ Mutant $\left(\right.$ Asahl $1^{\mathrm{P} 361 \mathrm{R} / \mathrm{P} 361 \mathrm{R}}$, Hom Farber) and WT $A s a h 1^{+/+}$animals were obtained by mating Het Asahl $^{\mathrm{P} 361 \mathrm{R} /+}$ mice. Genotyping was performed as described previously. ${ }^{13}$ Mice were housed at the Animal Resource Centre at the University Health Network (Toronto, Ontario, Canada) under specific pathogen-free conditions. All animal procedures were approved by the University Health Network Animal Care Committee.

\section{Behavioral Testing}

Voluntary locomotor and exploratory activities along with assessments of anxiety-like behavior were tested in an openfield arena. Mice were further profiled for a spectrum of behavioral activities by observation. Muscle strength and motor coordination abilities were assessed by a grip test and an accelerating rotating rod (rotarod) test, respectively. Open field, rotarod, and marble burying tests were performed in animals aged 7 to 8 weeks, and activity observations were collated from 9-week-old mice. For the grip test, mice were evaluated repetitively at 5,7 , and 9 weeks of age. At 7 weeks, the sequence of tests was as follows: open field on day 1 , rotating rod on day 3 and 4, and marble burying test on day 4 .

During testing, circadian rhythms were observed, with all test trials performed between $8 \mathrm{AM}$ and $1 \mathrm{PM}$. Nontest-related variability was reduced by using the same biosafety cabinet in a closed room. Experimenter variability was reduced by having the same person perform each trial of a given test on all animals. Data from any animal disturbed by an outside interference during testing were excluded from the analyses. An automatic tracking system (TrueScan, Coulbourn Instruments, Whitehall, PA) was used to record the total ambulatory movement time and distance traveled, the number of rears, and the number of entries into the center zone $(18.4 \times 18.4 \mathrm{~cm})$ of the arena. The last parameter was used as a measure of thigmotaxis, which is a tendency for mice to stay close to the walls of the testing apparatus because of anxiety induced by the novel environment.

For activity profiling, each mouse was observed in its home cage for 5 minutes, and an experimenter (C.K.D., blinded to the genotype) noted what activity (sniffing, rearing, locomotion, grooming, freezing, chewing, or burrowing) was being performed every 30 seconds for $5 \mathrm{mi}-$ nutes. ${ }^{15}$ In the marble burying test that we used, ${ }^{16}$ mice were also first habituated in their home cage and then transferred to a clean cage $(29.5 \times 17.5 \times 12.5 \mathrm{~cm})$ with $5 \mathrm{~cm}$ of bedding and 20 clean, identical navy blue marbles $(1.2 \mathrm{~cm}$ diameter) in a $4 \times 5$ matrix in two-thirds of the cage. Every 
mouse was placed in the one-third of the cage with no marbles and the lid was closed. After 30 minutes, the mouse was removed and the number of marbles buried was recorded. Marbles were considered buried when covered $>50 \%$ with bedding. For grip testing, mice were placed on metal mesh that was boxed in by smooth metal walls. ${ }^{17}$ This box was slowly turned upside-down, allowing mice to hold on. The time to fall was recorded to a maximum of $6 \mathrm{mi}-$ nutes. At each age, mice were tested three times with a 10minute rest between the trials. The mean of the three trials was used for statistical analyses. For rotarod testing, mice were placed on the device, which was then accelerated from 4 to $40 \mathrm{rpm}$ for 5 minutes. The time when mice fell off the rotating rod was automatically recorded. Animals were tested three times on 2 consecutive days.

\section{Collection and Processing of the Brains}

For magnetic resonance imaging (MRI), light microscopy, and electron microscopy (EM) studies, 3-, 5-, 7-, and 9-weekold mice were anesthetized and perfused transcardially with phosphate-buffered saline followed by $4 \%$ paraformaldehyde. The extracted brains were further immersion fixed overnight in $4 \%$ paraformaldehyde at $4^{\circ} \mathrm{C}$ and then transferred to and stored in phosphate-buffered saline at $4^{\circ} \mathrm{C}$.

For liquid chromatography-mass spectrometry (LC-MS) and mass spectrometry imaging (MSI), brains were isolated from 9-week-old euthanized male mice and divided along the midline sagittal plane. Half of the brain was frozen on dry ice for bulk LC-MS analyses and the other in liquid nitrogen in the vapor phase for MSI. Details of the consecutive tissue processing for individual types of analyses are provided in the following methodologic sections.

\section{Lipid Analysis by LC-MS}

For LC-MS, the complete half-brains were homogenized in $0.25 \mathrm{~mol} / \mathrm{L}$ sucrose (to get a concentration of $20 \%$, w/v) using the Fast-Prep apparatus (MP Biomedicals, Santa Ana, CA) and green-capped tubes (Lysing Matrix D). Protein concentrations were determined with bovine serum albumin as a standard using a bicinchoninic acid protein determination kit (Thermo Fisher Scientific, Waltham, MA) according to the manufacturer's instructions. Sphingoid bases were measured by LC-triple quadrupole mass spectrometer as previously reported. ${ }^{18}$ Other lipids were analyzed by ultraperformance liquid chromatography coupled to time of flight or ultraperformance liquid chromatography coupled to Exactive, both high-resolution mass spectrometers, as previously described. ${ }^{19}$ Three brains were analyzed for each genotype.

\section{MSI}

For MSI, frozen brain halves were cut into sections sagittally $(10 \mu \mathrm{m})$ in the 0.24 to 0.48 lateral range ${ }^{20}$ and analyzed by matrix-assisted laser desorption ionization (MALDI) MSI as previously described. ${ }^{21}$ Briefly, tissue samples were mounted on indium tin oxide-coated slides and sprayed with 2,5dihydroxybenzoic acid matrix. MALDI spectra were acquired across the entire tissue section on a Solarix dual source 7-T FT-ICR mass spectrometer (Bruker Daltonics Inc., Billerica, MA) to detect the lipid species of interest in positive ion mode $(\mathrm{m} / \mathrm{z}=200$ to 1200$)$ or negative ion mode $(\mathrm{m} /$ $\mathrm{z}=200$ to 2000) with a SmartBeam II laser operating at $1000 \mathrm{~Hz}$, a laser spot size of $25 \mu \mathrm{m}$, and a raster width of 200 $\mu \mathrm{m}$ for general profiling or $75 \mu \mathrm{m}$ for high-resolution images. Images were generated using FlexImaging software version 4.0 (Bruker Daltonics) and structures confirmed using an inhouse database of FD Cer as previously described. ${ }^{21}$ Hematoxylin and eosin (H\&E) staining of the brain sections was performed after the MALDI-MSI imaging. Three brains were analyzed for each genotype.

\section{MRI}

Fixed mouse brains (5-, 7-, and 9-week-old) were immersed in Fomblin and scanned using a 9.4-T Varian Direct Drive animal MRI/MRS system (Agilent Technologies Inc., Santa Clara, CA) with a 14-mm i.d. single loop receiver coil (Doty Scientific Inc., Columbia, SC) and a 7-cm i.d. $1 \mathrm{H}$ transmission coil (m2m imaging Co., Cleveland, $\mathrm{OH}$ ). T2-weighted images $\left(78 \times 78 \times 500 \mu \mathrm{m}^{3}\right.$, no gap) were acquired using a fast spin echo sequence (repetition time/ echo time $=5000 / 44 \mathrm{~ms}$; echo train length $=4$; number of excitations $=8$ ). Image sequences were evaluated and individual images were extracted for figure preparation in Medical Imaging Processing and Visualization software version 7.0.1 (NIH, Bethesda, MD). One brain was analyzed for each genotype and age group.

\section{Histopathologic Analysis, Antibodies, Immunohistochemistry, and Immunofluorescence}

Cerebra and cerebella from Hom Farber and WT mice aged $3,5,7$, and 9 weeks were embedded in paraffin, cut into sections (coronally in cerebrum and sagittally in cerebellum) at 5 to $8 \mu \mathrm{m}$, and stained according to the standard $\mathrm{H} \& \mathrm{E}$, periodic acid-Schiff (PAS), and Luxol fast blue (LFB) protocols. Immunohistochemistry (IHC) detection of cathepsin D (CathD) was performed using a monoclonal rabbit antibody (1:500, LifeSpan Biosciences, Seattle, WA) on 2- to 5- $\mu \mathrm{m}$-thick cerebral and cerebellar sections. An anti-mouse Dako Envision kit (Dako, Glostrup, Denmark) was used for secondary detection according to the manufacturer's instructions. For immunofluorescence labeling, paraformaldehyde-fixed brains were cut sagittally in the midline and then divided into cerebral and cerebellar with brain stem parts by a single coronal cut at the mesencephalic level. The separated fragments were embedded into $8.0 \%$ sucrose and 3.5\% agarose. Serial sections $(35-\mu \mathrm{m}$ thick) cut coronally in the cerebrum (bregma -0.58 to -3.88 ) and 
sagittally in the cerebellum (lateral 0.00 to 1.32 ) were cut using a Leica VT-1000S vibratome (Leica Microsystems, Wetzlar, Germany). Sections corresponding to the bregma -1.94 to -2.30 and lateral 0.40 to 0.84 ranges $^{20}$ were selected from the cerebra and cerebella, respectively. Matched sections were stained by multilabeling immunofluorescence protocols ${ }^{22}$ using the following primary antibodies and dilutions: mouse anti-neuronal nuclei (NeuN) monoclonal antibody (1:1000, Chemicon, Temecula, CA), mouse anti-calbindin monoclonal antibody D-28K (1:3000, Sigma-Aldrich, St Louis, MO), rabbit anti-calbindin polyclonal antibody (1:800, Chemicon), rat anti-CD68 monoclonal antibody (1:1000, AbD Serotec, Kidlington, UK), mouse anti-glial fibrillary acidic protein (GFAP) monoclonal antibody G-A-5 (1:3000, Sigma-Aldrich), and mouse anti-neurofilament medium chain monoclonal antibody (1:500, Novus Biologicals, Littleton, CO). Species-specific detection of the primary antibodies used secondary antibodies conjugated to Alexa Fluor (AF) 488 and 546 dyes (Invitrogen, Carlsbad, CA). A minimum of two to three male brains per genotype and age group were analyzed.

\section{Light Microscopy}

H\&E-, PAS-, LFB-, and IHC-stained tissue sections were imaged using Olympus AX70 (Olympus, Tokyo, Japan) and Nikon E800 (Nikon, Tokyo, Japan) microscopes with MagnaFire or Olympus DP70 cameras, respectively. As previously described, ${ }^{23}$ an Olympus IX70 microscope with $\mathrm{HQ}_{2}$ camera (Photometrics, Britannia, AZ), Proscan II $x y z$ stage (Prior Scientific, Rockland, MA), and SmartSutter 10 position filter wheel shutters (Sutter, Novato, CA) were used to serially $x y$ image multifluorescently labeled sections. Exposure time for all antibody combinations and channels was $750 \mathrm{~ms}$ (gain $2 \times$ ). Excitation/emission conditions for the AF488 were 490/20/beam splitter 480 to 513/em.535/40, and for AF546 the conditions were 572/23/beam splitter 555 to $588 / 630 / 60 \mathrm{~nm}$. Spatially overlapping, double-channeled, 14bit images were acquired with the Plan $10 \times$ (numerical aperture 0.25 ) objective, down-sampled to 8 bits, and digitally stitched in Metamorph/MetaFluor version 7.8 (Molecular Devices, Sunnyvale, CA) and Photoshop CS6 version 13.0.1 $\times 64$ (Adobe, San Jose, CA) softwares. To gain higher optical resolution, immunofluorescence-labeled sections were imaged by a laser scanning confocal microscope Zeiss Meta Duo V2 (Zeiss, Oberkochen, Germany) using either Plan AF $63 \times$ (numerical aperture 1.4) or Plan AF $20 \times$ (numerical aperture 0.40) objectives. Z-stack image data sets are presented as maximum intensity projections.

\section{Electron Microscopy}

After collecting serial vibratome sections for light microscopy analyses, a 250-mm-thick single section was cut from the cerebrum and cerebellum of 3- and 9-week-old FD mice and controls. The following anatomical regions (nomenclature and abbreviations correspond to Franklin and Paxinos $^{20}$ ) were dissected into separate tissue blocks and were processed for EM as previously reported $^{23,24}$ : neocortex and hippocampus [primary somatosensory cortex, primary and secondary visual cortex (lateral, mediolateral and mediomedial areas), CA1-CA3 hippocampal fields]; internal capsule, hippocampal fimbria, thalamus (ventral posteromedial and posterolateral thalamic nucleus), striatum, dorsal lateral geniculate nucleus, and lateral ventricle; and cerebellar lobules 1 to 5 and medial deep cerebellar nuclei. Thin sections were imaged using Philips CM10 electron microscope (Philips Electron Optics, Eindhoven, the Netherlands). Individual CNS cell types were identified based on their morphologic characteristics (neurons, astrocytes, and microglia) as described by Peters et $\mathrm{al}^{25}$ and oligodendrocytes as described by Fletcher et al. ${ }^{26}$ Categorization of the vascular organization into endothelial cells, pericytes, and perivascular macrophages follow the work of Guillemin et al. ${ }^{27}$

\section{Statistical Analysis and Figure Preparation}

Statistical analyses of the open field, marble burying, and activity observation assays were assessed by an unpaired $t$-test in GraphPad Prism software version 5 (GraphPad Software, Inc., La Jolla, CA). For the grip test and rotarod assays, significance was assessed by repeated-measures analysis of variance followed by $t$-tests performed in JMP statistical software version 11 (SAS Institute Inc, Cary, NC). Significance in biochemical and weight studies was assessed by one-way analysis of variance or two-way analysis of variance, respectively, with Bonferroni posttests in GraphPad Prism software version 5. Graphs were plotted in GraphPad Prism software version 5. Figures were prepared in Photoshop CS6 (Adobe, San Jose, CA). For better contrast and visibility and without altering the biological information, images were stretched to fill the full dynamic 8-bit ranges. Neuroanatomical nomenclature and abbreviations used in the manuscript correspond to the work of Franklin and Paxinos. ${ }^{20}$

\section{Results}

Expression of a Complex Behavioral Phenotype in Asah1 $^{\text {P361R/P361R }}$ (Hom Farber) Mice

Hom Farber mice replicate many of the phenotypic features of FD patients. ${ }^{13}$ To delineate gross behavioral and neurologic consequences of ACDase deficiency in this model, we first compared Hom Farber mice with WT mice in their ambulation and exploration abilities, their compulsive or stereotypic and anxiety behaviors, and their muscle strength and motor coordination capabilities. In the open-field test, Hom Farber mice (7 to 8 weeks old) spent less time ambulating (Figure 1A) and covered half as much distance (Figure 1B). They also had one-fifth of the rearing 

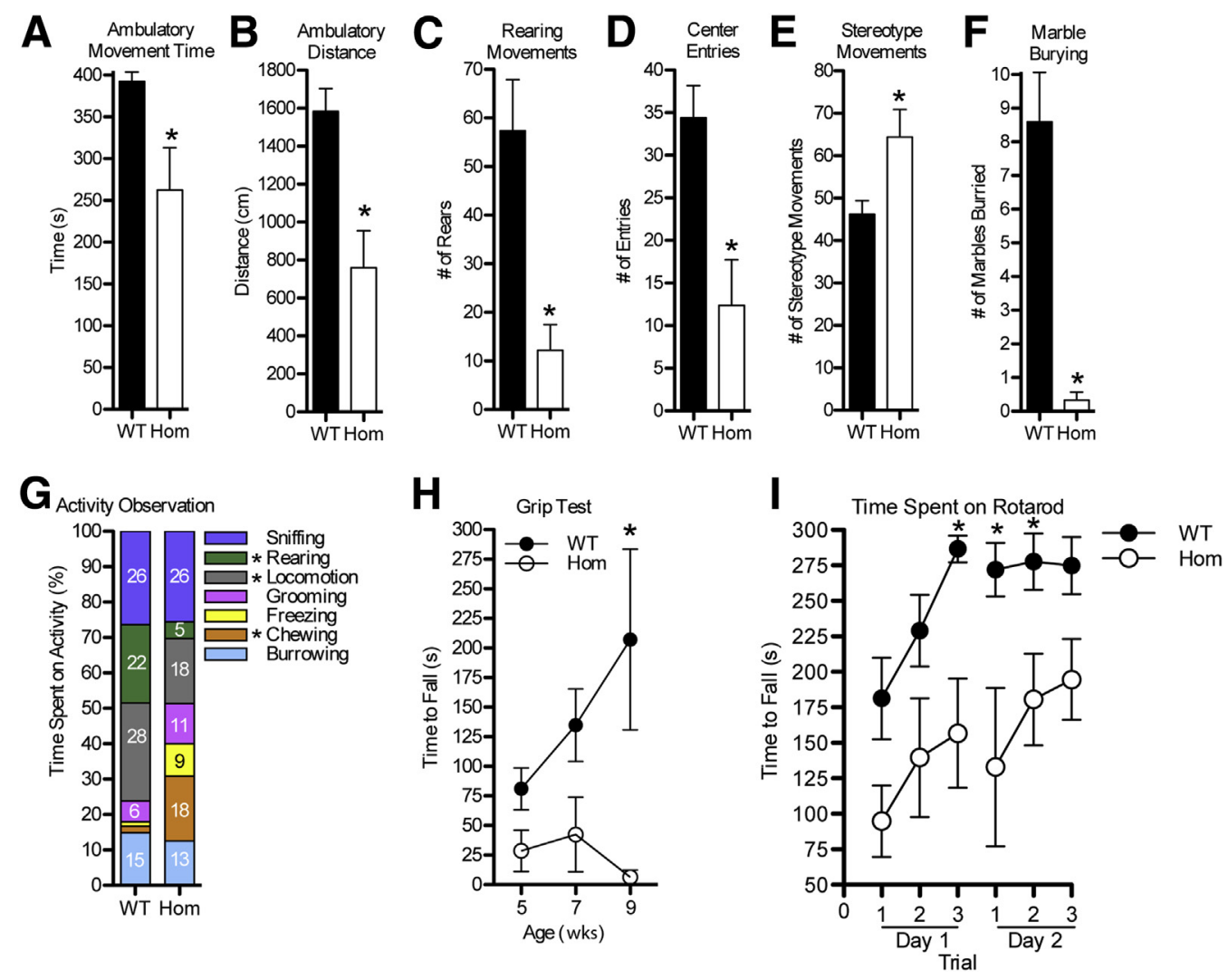

Figure 1 Homozygous (Hom) Farber mice have decreased locomotor and exploration activity, increased thigmotaxis, and perform poorly in grip strength and rotating rod (rotarod) tests. A-E: The activity of 7- to 8-week-old wild-type (WT) and Hom mice in an open-field arena was recorded for 10 minutes. A-C: Hom mice had decreased voluntary locomotion and rearing. D: Hom mice had increased thigmotaxis (tendency to stay close to the walls of the open-field apparatus) behavior by entering the center zone of the arena less frequently. $\mathbf{E}$ and $\mathbf{F}:$ 7- to 8-week-old Hom mice had increased stereotype behavior on the open-field test but decreased stereotype behavior on the marble burying test. G: 9-week-old Hom mice spent a greater percentage of their time chewing compared with WT animals and less time on locomotion and rearing. H: Hom mice underperformed in the grip test; when the same mice were subjected to the grip test at 5, 7, and 9 weeks of age, Hom mice performed poorer at 9 weeks of age. Neither WT nor Hom mice significantly increased the length of time for which they can grip over time. I: Hom mice fell off more quickly on an accelerating rotarod. Both WT and Hom mice were able to learn over trials and maintain that ability to the second day of testing. All data are presented as means \pm SEM. Male and female animals were combined for all tests and analyses. $n=8$ WT mice and 5 Hom mice for the open-field test $(\mathbf{A}-\mathbf{E}) ; n=10$ WT mice and 9 Hom mice for the marble burying test (F); $n=13$ WT and 12 Hom mice for activity observation (G); $n=6$ WT mice and 5 Hom mice for the grip test $(\mathbf{H}) ; n=8$ WT mice and 4 Hom mice for the rotarod test $(\mathbf{I})$. ${ }^{*} P<0.05$ in the open-field, marble burying, and activity observation as assessed by an unpaired $t$-test and for the grip test and rotarod test as assessed by repeated-measures analysis of variance followed by $t$-tests.

movements of controls (Figure 1C), which is also considered a measure of exploratory activity. As well, Hom Farber mice had an increased thigmotaxis behavior by entering the center zone one-third as frequently (Figure 1D) and increased stereotypic behavior in the open-field analysis (back and forth movements in the corners of the arena) (Figure 1E). Although the marble burying test (performed in 7- to 8-week-old mice) results appear to oppose the openfield test results and actually suggest a reduced stereotypic behavior (Hom Farber mice buried fewer marbles) (Figure 1F), it is more likely that Hom Farber mice buried fewer marbles because they ambulate less (Figure 1B).

Observation-based activity assessments (performed in 9-week-old mice) also had significant differences between Hom Farber mice and WT mice. Hom Farber mice spent less time rearing and locomoting and spent more time chewing (Figure 1G). This finding is consistent with the results of the open-field test. Next, the strength of the mice was assessed biweekly using an inverted grip test. Testing began at 5 weeks of age, and the same mice were tested every 2 weeks. WT and Hom Farber mice performed similarly when first tested at 5 weeks of age, but by 9 weeks of age Hom Farber mice performed significantly worse than WT mice (Figure 1H). Neither genotype had significant improvements over time. Finally, Hom Farber mice presented with impaired motor coordination, falling more quickly off the rotarod (Figure 1I). Both genotypes significantly improved on each trial, yet Hom Farber mice still fell off more quickly than WT mice.

\section{Abnormal Sphingolipid Profiles in the Brains of Hom Farber Mice}

The total sphingolipid profile of WT, Het, and Hom Farber mouse brains was analyzed by LC-MS. As a family, Cer, hydroxy-ceramides (Cer-OH), dihydroceramides (dhCer), 
Sph, dihexosylceramides (DHC), and monosialodihexosylganglioside (GM3) concentrations were all elevated in Hom Farber mice compared with WT mice by absolute values and by fold-increases over WT levels (Supplemental Figure S1A and Figure 2A). No significant changes in sphingolipid profiles were seen in Het animals compared with WT animals. In Hom Farber mice, the elevations resulted in altered brain sphingolipid composition: Cer, Cer-OH, dhCer, DHC, and GM3 ganglioside constituted a significantly greater percentage of total brain lipids, with a concomitant reduction in the proportion of monohexosylceramides (MHC) and sphingomyelin (SM) (Figure 2B). The change in distribution due to increased $\mathrm{Cer}$ and $\mathrm{Cer}-\mathrm{OH}$ levels was a consequence of every chainlength isoform of these classes being elevated (Figure 2, C and E, Supplemental Figure S1, B and C) but to different degrees (Figure 2, D and F). Cer-OH was the primary class of sphingolipids elevated in brains, increasing by $>100$-fold in Hom Farber mice (Figure 2A). All Cer-OH chain-length isoforms contributed to this elevation, each being increased $>40$-fold (Figure 2E). Similarly, all isoform species of dhCer were elevated (Supplemental Figure S1D, Figure 2, G and $\mathrm{H}$ ), resulting in the dhCer contribution to brain lipid composition tripling from $0.05 \%$ to $0.18 \%$ (Figure $2 \mathrm{~B}$ ). Sph content greatly exceeded dihydrosphingosine content (dhSph, or sphinganine) in WT and Het mice; this difference was further pronounced in Hom Farber mice (Supplemental Figure S1E, Figure 2, I and J). Interestingly, whereas nearly all DHC species (Figure $2 \mathrm{O}$ and Supplemental Figure S1H) as well as the GM3 ganglioside (Figure 2S, Supplemental Figure S1J, and Supplemental Figure S2E) were elevated in Hom Farber animals, most MHC chain lengths were unchanged [only 16:0 was elevated (Figure 2, K and L, and Supplemental Figure S1F) resulting in a reduction in MHC as a brain lipid class (Figure 2B)]. Hydroxymonohexosylceramides (MHC-OH) were the only lipid class measured that did not have any species lengths changed (Figure 2, $\mathrm{M}$ and $\mathrm{N}$, and Supplemental Figure $\mathrm{S} 1 \mathrm{G}$ ), and its proportion in brain was not altered (Figure 2B). Finally, most SM species were unchanged, and the ones changed were altered to a lesser fold than other sphingolipid classes (Figure 2, Q and R, and Supplemental Figure S1I), resulting in the overall SM proportion being decreased in global sphingolipid composition (Figure 2B).

\section{MALDI-MSI Visualization of Sphingolipid Accumulation Shows Regional Accumulation of Selected Species}

MALDI-MSI allows the identification and mapping of biomolecules directly in tissues; a previous study using this method characterized Cer accumulation in kidneys from the same Hom Farber mouse model. ${ }^{21}$ To complement the quantitative LC-MS data obtained for the different Cer species in Hom Farber brain tissues, MALDI-MSI was used to determine the anatomical locations of selected Cer and ganglioside species in the same brains used for
LC-MS. Two of the most abundant Cers detected by LC-MS in Hom Farber brain were $\operatorname{Cer}(\mathrm{d} 18: 1 / 16: 0)$ and Cer(d18:1/18:0), and images of their distribution in sagittal sections of Hom Farber and WT brains are shown in Figure 3. In the Hom Farber brains, Cer(d18:1/16:0) was primarily localized in the cerebellar granule cells layer, cerebral cortex, and paraventricular and medial thalamic nuclei (Figure 3) and strikingly absent in cerebellar white matter (WM). In contrast, localization of $\operatorname{Cer}(\mathrm{d} 18: 1 / 18: 0)$ in FD brain was increased in septal nuclei and corpus callosum. An overlay image of the two Cers highlights these different distributions (Figure 3), with their co-localization in cerebellar granule cells layer, and the predominant $\operatorname{Cer}(\mathrm{d} 18: 0 /$ 16:0) midbrain and $\operatorname{Cer}(\mathrm{d} 18: 1 / 18: 0)$ cerebral cortex accumulations. The distribution of a third species, $\operatorname{Cer}(\mathrm{d} 18: 1 /$ 20:0), partially overlapped with that of $\operatorname{Cer}(\mathrm{d} 18: 1 / 18: 0)$ and Cer(d18:0/16:0) but was not as widespread (Figure 3). The representative data for these three Cers indicate that their distributions in the Hom Faber brains are localized to specific brain regions and can be species different.

The most abundant ganglioside and sulfatide species were also measured in negative ion mode in the same sagittal sections (Supplemental Figure S2, B-E). Shown are the monosialotetrahexosylganglioside (GM1)-, monosialotrihexosylceramide (GM2)-, GM3-, and sulfatidecontaining Cer(d18:1/18:0). In Hom Farber brain, the GM1 content was elevated in the cerebral cortex compared with the WT brain, and GM2 and GM3 concentrations were elevated in the cerebellar granule cells layer, septal nucleus, and cerebral cortex. The sulfatide concentration was elevated in the cerebellar and cerebral (corpus callosum) WM and the midbrain (except the septal nuclei) (Supplemental Figure S2B). Interestingly, the Cer(d18:1/18:0)-containing GM2 and GM3 co-localized with the corresponding accumulated Cer in the Hom Farber brains (Figure 3).

Hydrocephalus, Extensive Tissue Pathologic Findings, and Subcellular Storage Changes in a Broad Range of CNS Cell Types in Hom Farber Mice

\section{Gross Brain Pathologic Findings}

Hydrocephalus was previously detected in approximately $70 \%$ of 10 -week-old Hom Farber mice by MRI. ${ }^{13}$ In agreement with the latter study and to further explore initiation and temporal progress of this abnormality, in the current study we performed MRI on fixed brains of Hom Farber mice and WT mice aged 5, 7, and 9 weeks. Hydrocephalus that affected all ventricles (lateral, third, and fourth) was evident in a few Hom Farber mice already at 7 weeks (Figure 4A, Supplemental Figure S3A).

The additional fluid in the brains of Hom Farber mice contributed to an increase in brain weight relative to total body weight (Figure 4B). The absolute weight of Hom Farber brains was similar to that of WT and Het mice at ages 5, 7, and 9 weeks (Supplemental Figure S3B), yet Farber mice weigh less than WT and Het mice. ${ }^{13,14}$ Therefore, the 

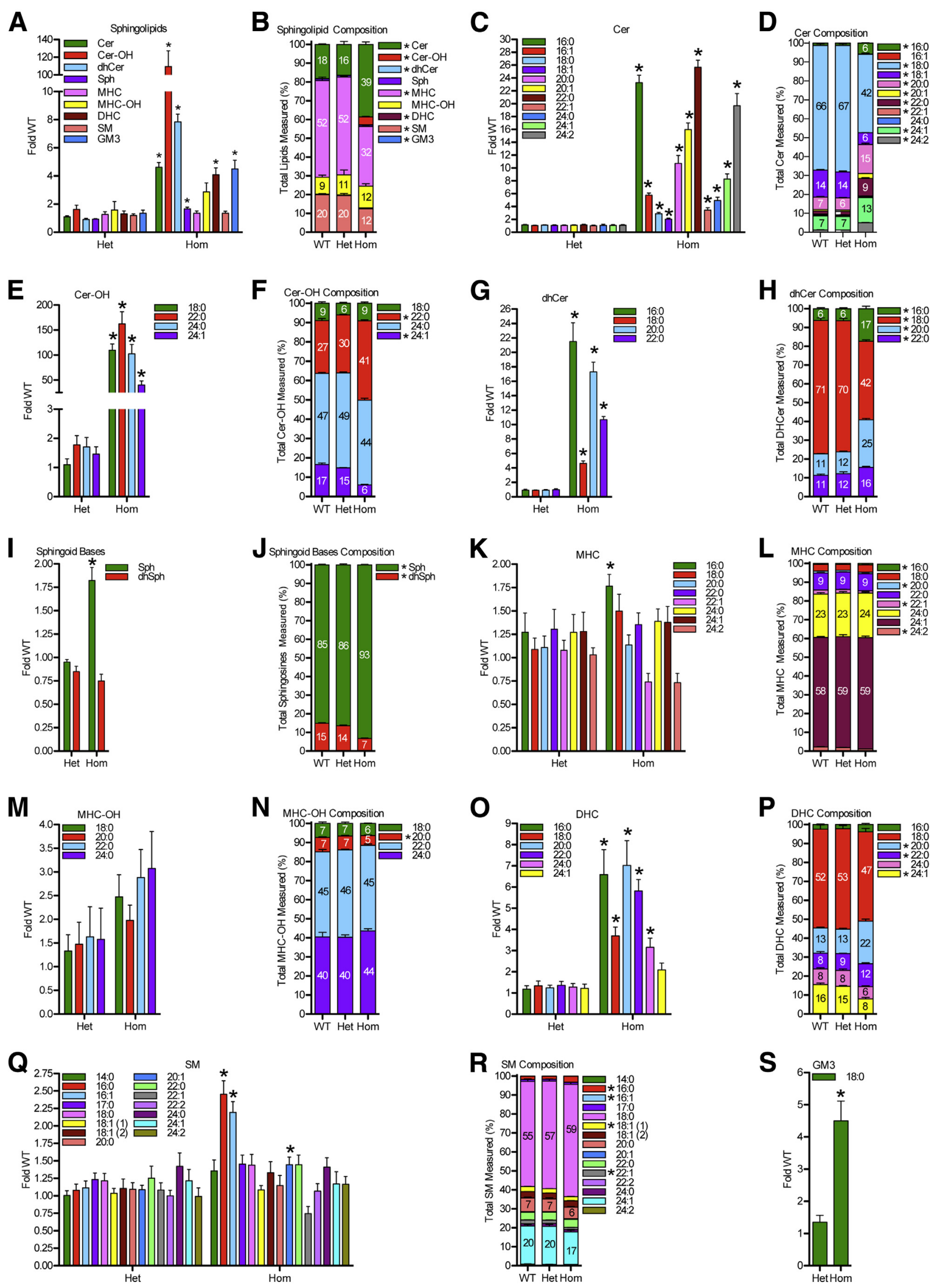
similarly sized brain constitutes double the percentage of total body weight of Hom Farber mice.

Because of the fragility of the hydrocephalic brains, we primarily focused further neurohistologic and ultrastructural studies on nonhydrocephalic Hom Farber brains.

Cerebral and Cerebellar (Immuno) Histopathologic Analysis We analyzed cerebral and cerebellar histopathologic findings in H\&E, PAS, LFB, and CathD immunohistochemically stained sections from 3-, 5-, 7-, and 9-week-old Hom Farber and WT mice. Given the limited comparative data on the spatial distribution and cellular and tissue nature of the human FD CNS pathologic changes, 5,6 we decided to focus our studies at cerebral and cerebellar areas previously reported as preferentially affected in murine models of other lysosomal storage disorders. ${ }^{28-32}$ The cerebral volume that we analyzed on coronal sections is highlighted by red arrows in Figure 3 and contains the primary somatosensory cortex, hippocampus, ventral posteromedial nucleus, ventral posterolateral nucleus, posterior group of thalamic nuclei, and subcortical WM (internal capsule included). In cerebellum, we tested the tissue volume that was laterally limited by the extent of the lobule X.

We found no profound neuronal migration abnormalities in sections from Hom Farber mice. That said, we could identify evidence of lysosomal abnormalities by CathD IHC in specific cerebral and cerebellar neuronal populations in Hom Farber mouse brains already at 3 weeks despite the fact that major neuronal cytoplasmic vacuolization was not discernible on a global scale even at 9 weeks (Supplemental Figure S4, A and B). As a dominant feature, we found numerous abnormal and variably slightly $\mathrm{PAS}^{+}$cells with expanded foamy cytoplasm both in the cerebra and cerebella of Hom Farber mice. At 3 weeks of age, these cells already appeared in the subcortical WM. The regions specifically affected were the external capsule and internal capsule and hippocampal fimbria regions. Later ( $>5$ weeks), these cells started forming granuloma-like accumulations and frequently organized into perivascular cuffs. Other areas populated by these cells were the meninges and to a lesser extent the stroma of choroid plexi of all cerebral ventricles. Contrary to what was observed in WM, in specific gray matter (GM) areas these cells accumulated into discrete and sparsely distributed perineuronal clusters.

To delineate the cell type-specific pathologic abnormalities, we co-stained sections from Hom Farber and WT mouse brains with anti-NeuN, anti-CD68, and anti-GFAP primary antibodies to highlight neurons, microglia and/or macrophages, and astrocytes, respectively (Figure 5).
Similar to the observations in the histologic stains, NeuN staining suggested that the gross overall neuronal density and organization of the cortical and subcortical GM was not substantially affected at any age tested.

Abnormal $\mathrm{CD}^{+} 8^{+}$microglia and/or macrophages, which corresponded to the abnormal and variably slightly $\mathrm{PAS}^{+}$cells in the WM and GM, represented the major pathologic finding in Hom Farber mice. Contrary to the coalescing granuloma-like accumulations in WM (Figure 5 and Figure 6, A and B), $\mathrm{CD}^{+} 8^{+}$cells formed well-discernible neuronophagic clusters in specific GM regions. Within the analyzed cerebral volume, the GM areas affected by the latter pathologic finding were the CA1 region of hippocampus (Figure 5 and Figure 6C), layer II of the primary somatosensory (S1) cortex (Figure 5 and Figure 6D), and the posterior group of thalamic nuclei and the thalamic ventral posteromedial nucleus (data not shown). Similar to the $\mathrm{CD}^{+} 8^{+}$microglial and/or macrophage pathologic findings, $\mathrm{GFAP}^{+}$gliosis was initiated in the WM and only later appeared in the GM (Figure 5). A comparison with abnormalities in a brain of a 9-week-old animal affected by hydrocephaly $\left(\mathrm{GFAP}^{+}\right.$ abnormality is particularly accentuated) is provided in the Supplemental Figure S3C.

We also performed LFB staining to highlight any major abnormality in WM formation and/or myelination. At 3 weeks of age, the cerebral subcortical WM appeared thinner in Hom Farber mice when compared with WT littermates. In 5- to 9-week-old Hom Farber animals, the abnormal build-up of $\mathrm{CD}^{+} 8^{+}$microglial and/or macrophage granuloma-like accumulations was the dominant WM pathologic findings (Figure 5 and Figure 6, A and B). Anti-neurofilament medium chain antibody and immunofluorescent staining was used to examine axonal bundles in the WM, but this did not reveal significant neuroaxonal dystrophy in Hom Farber mice (data not shown).

Cerebellar pathologic findings were, in general, similar (Figure 7A) to the abnormalities observed in the cerebra of Hom Farber mice. However, even in 9-week-old animals, the density of calbindin ${ }^{+}$Purkinje cells (PCs) was comparable in all the cerebellar zones and lobules between Hom Farber mice and WT mice. Correspondingly the thickness of the molecular layer was also not substantially reduced in any of the cerebellar zones and/or lobules. Furthermore, by immunofluorescence we did not detect either notable abnormalities of calbindin $^{+}$or neurofilament medium chain $^{+} /$calbindin $^{-}$axons (data not shown) or substantial morphologic alterations of PC dendritic arbors (Figure 7B and Supplemental Figure S4B).

\footnotetext{
Figure 2 Specific sphingolipid species are altered in homozygous (Hom) Farber mice. Sphingolipids in the brain of 9-week-old wild-type (WT), heterozygous (Het), and Hom mice were measured by liquid chromatography-mass spectrometry. A: Fold elevation over WT for each sphingolipid class measured. B: The percent composition of the various sphingolipid classes measured. D, F, H, J, L, N, P, and R: Fold elevation over WT of individual isoforms within each sphingolipid class. C, E, G, I, K, M, 0, Q and S: Each isoform chain length is shown in percent relation to other chain lengths in its respective sphingolipid class. All data are presented as means \pm SEM. $n=3$ animals for each genotype. ${ }^{*} P<0.05$ in a 1-way analysis of variance with Bonferroni posttests for WT versus Hom mice. Cer, ceramide; $\mathrm{Cer}-\mathrm{OH}$, hydroxy-ceramide; dhCer, dihydroceramide; $\mathrm{DHC}$, dihexosylceramide; dhSph, dihydrosphingosine; GM3, monosialodihexosylganglioside; MHC, monohexosylceramide; $\mathrm{MHC}-\mathrm{OH}$, hydroxy-monohexosylceramide; SM, sphingomyelin; Sph, sphingosine.
} 

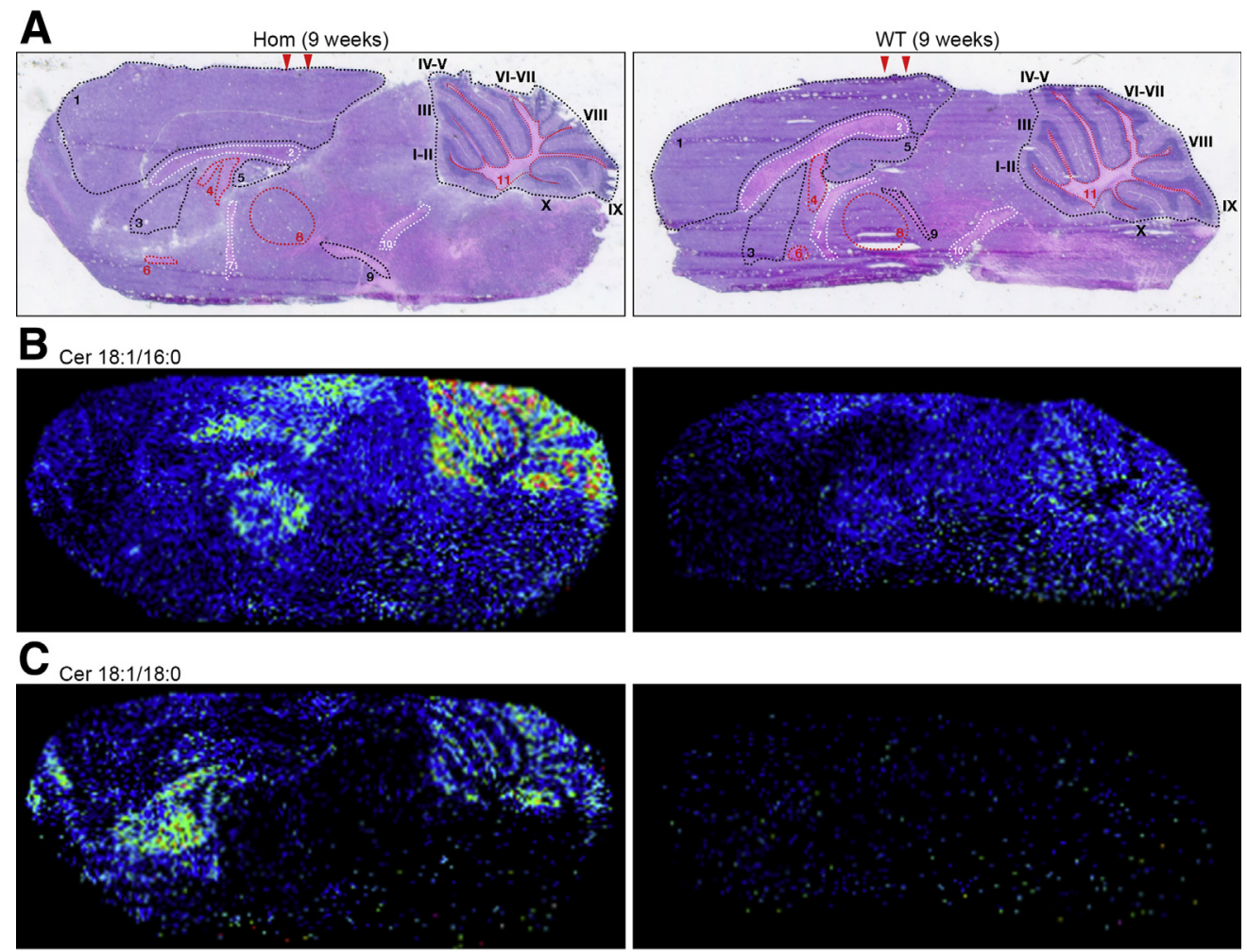

D $_{\text {Cer } 18: 1 / 16: 0 \&}$
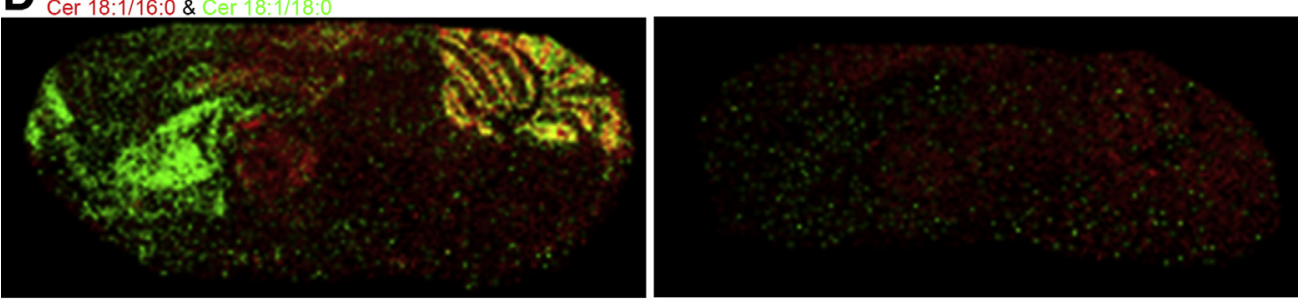

E $\operatorname{Cer} 18: 1 / 20: 0$
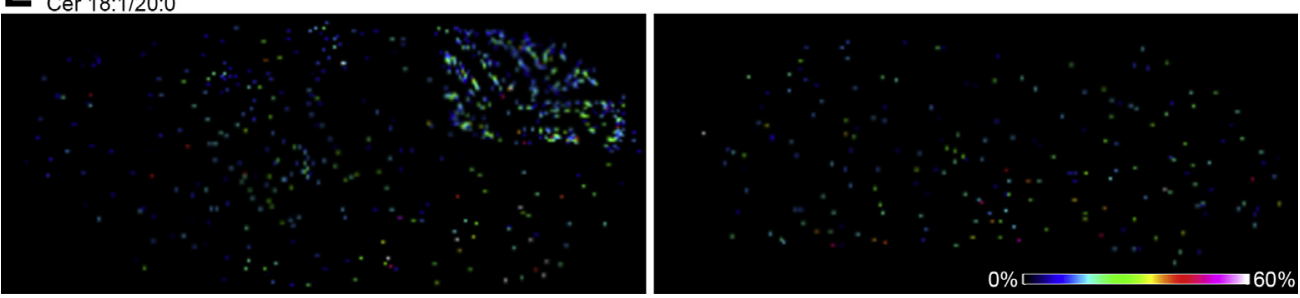

Figure 3 Matrix-assisted laser desorption ionization mass spectrometry imaging (MALDI-MSI) analysis reveals selective distribution of ceramides by specific brain region in 9-week-old homozygous (Hom) Farber mice. A: Sagittal tissue sections of Hom Farber and wild-type (WT) brains were analyzed by MALDI-MSI in positive ion mode $(\mathbf{B}-\mathbf{E})$ as previously described. ${ }^{21}$ Red arrowheads limit the approximate brain volume from which the coronal sections shown in Figure 5 were imaged. Shown are the localizations for ceramide $(\operatorname{Cer})(\mathrm{d} 18: 1 / 16: 0)+$ potassium $(\mathrm{m} / \mathrm{z}=576.561)(\mathbf{B}), \operatorname{Cer}(\mathrm{d} 18: 1 / 18: 0)+$ potassium $(\mathrm{m} / \mathrm{z}=604.496)(\mathbf{C})$, and $\operatorname{Cer}(\mathrm{d} 18: 1 / 20: 0)+$ potassium $(\mathrm{m} / \mathrm{z}=632.526)(\mathbf{E})$. D: An overlay image in the fourth panel is included for Cer(d18:1/16:0) (red) and Cer(d18:1/18:0) green, with overlapping regions appearing as yellow. The relative intensity levels of each species are represented as color pixels as indicated by the heatmap bars. MALDI-MSI data for specific ganglioside species were acquired in negative ion mode and are given in Supplemental Figure S2. Sections were stained with hematoxylin and eosin (H\&E) stain (A) after the MALDI-MSI imaging sequence for spatial determination, and anatomical regions of each brain are indicated as follows: 1 , cerebral cortex; 2 , corpus callosum; 3, septal nuclei; 4, dorsal fornix and ventral hippocampal commissure; 5, hippocampus; 6, anterior commissure; 7, stria medullaris thalami; 8, paraventricular and medial thalamic nuclei; 9 , fascicullus retroflexus; 10 , superior cerebellar peduncle; 11 , cerebellar WM; and I to X, cerebellar lobules. H\&E images are identical to H\&E images in Supplemental Figure S2. Cer 18:1/16:0 corresponds to Cer(d18:1/16:0), Cer 18:1/18:0 to Cer(d18:1/18:0), and Cer 18:1/20:0 to Cer(d18:1/20:0). Representative images are shown for a group of brains tested (three per genotype). Scale bar (heat map) $=5 \mathrm{~mm}$.

As in the cerebrum, the abnormal $\mathrm{CD} 68^{+}$microglia and/ or macrophages were the hallmark of the cerebellar pathologic findings in Hom Farber mice. At 3 weeks of age, we found foamy $\mathrm{CD}^{+} 8^{+}$cells in the cerebellar WM (data not shown). Their distribution, again, was closely spatially linked to brain vessels. Importantly, these CD68 ${ }^{+}$ cells did not preferentially populate the molecular layer of the anterior and/or posterior cerebellar cortical zones or the 

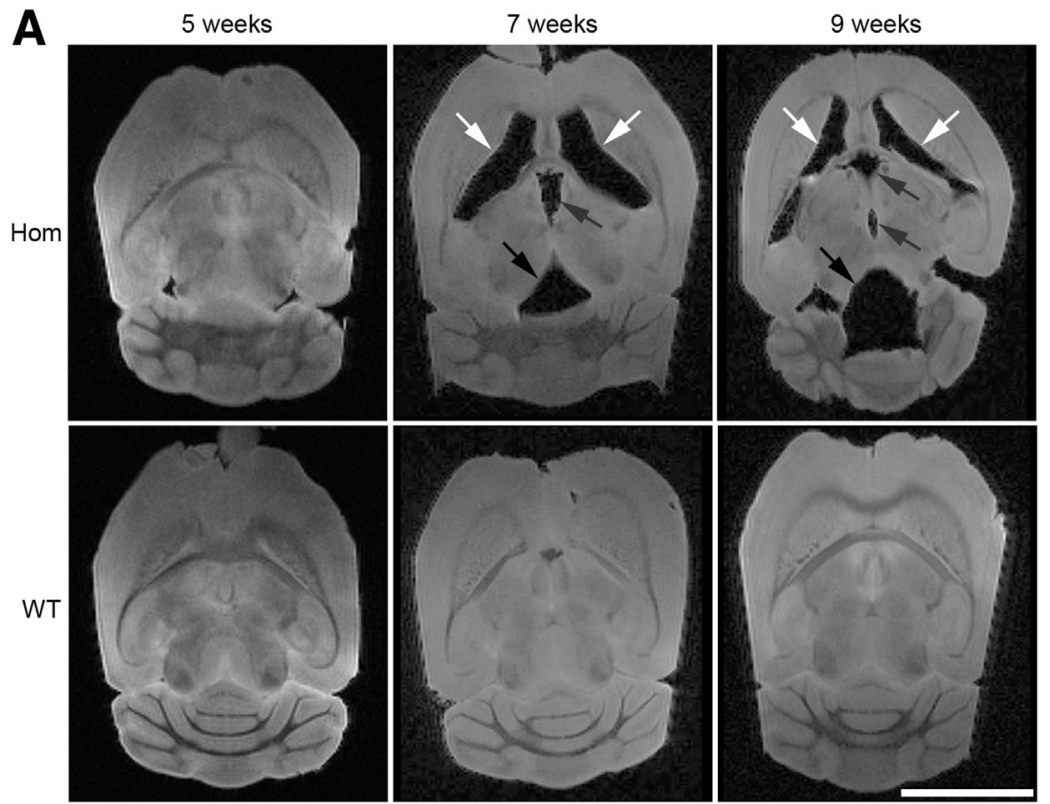

B

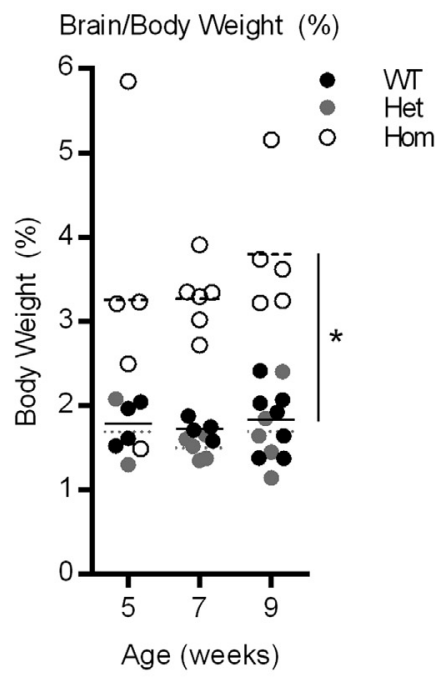

Figure 4 Hydrocephalus affects all ventricles in the brains of $A s a h 1^{P 361 R / P 361 R}$ homozygous (Hom) mice. A: Hydrocephalus of all ventricles (lateral - white arrows, third - gray arrows, and fourth - black arrows) is first detected in Hom mice by magnetic resonance imaging (MRI) (axial projection) at 7 weeks of age. B: The whole body and brains of 5-, 7-, and 9-week-old mice were weighed. Hom brains represent a greater percentage of the body. Means of wild-type (WT), heterozygous (Het), and Hom brain/body weights are indicated by solid, dotted, and dashed lines, respectively. Comparison to coronal brain MRI projections is provided by Supplemental Figure S2, A and C. $n=4$ to 7 WT mice, $n=2$ to 5 Het mice, $n=5$ to 6 Hom mice (B). ${ }^{*} P<0.05$ as assessed by a two-way analysis of variance with Bonferroni posttests (B). Scale bar $=5 \mathrm{~mm}(\mathbf{A})$.

deep cerebellar nuclei. However, at later ages, we found granuloma-like clustering of $\mathrm{CD}^{+} 8^{+}$cells within the cerebellar GM (PC layer included) and WM, subependymal zone of the fourth ventricle, and cerebellar meninges. Interestingly, the GFAP signal originating from astrocytes of the PC layer (Bergmann glia) was stronger in Hom Farber mice than in WT mice even in the absence of extensive and patterned PC degeneration in Hom Farber animals.

\section{Ultrastructural Pathologic Analyses}

We performed ultrastructural analyses on the brains of 3- and 9-week-old Hom Farber and WT mice to evaluate the character and progression of the subcellular storage pathologic features. Storage changes could be detected in a broad range of neuronal and nonneuronal cell types in brains from Hom Farber mice as early as 3 weeks of age; these were not found in the brains of their WT littermates. All storage patterns (CTBs; banana, spindle, or needle-like bodies; and zebra-like bodies) previously linked to FD were identified in Hom Farber mice. That said, the appearance of the storage bodies was variable between specific cell types. Likewise, individual cells often expressed a combination of several morphologic storage patterns.

The abnormal microglia and/or macrophages in the brain presented with dominant storage pathologic features. This presentation was comparable to observations made previously concerning liver and peripheral nerves of
Hom Farber mice. ${ }^{13}$ The confluent storage bodies in these cells had a finely granular matrix (Figure 8, A, C, and E) with occasionally embedded CTBs (Supplemental Figure S5A). Similar to findings under light microscopy, granuloma-like accumulations of storage-laden microglia and/or macrophages were frequently (but not exclusively) observed in WM. These developed in close vicinity to brain vessels (Figure 8, C and E). Moreover, a fraction of the cells contained within these abnormal clusters likely degenerated. As a result, we identified foci of accumulated cellular debris with embedded storage material (Supplemental Figure S5B) in older animals.

Unlike in 3-week-old Hom Farber mice (Figure 8B), in WM of 9-week-old Hom Farber animals, we found zebralike and/or cleared banana-like bodies in the cytoplasm of cells with oligodendroglial morphologic characteristics (Figure 8D). Zebra-like bodies could also be found in WM within the processes of cells with electron-dense cytoplasm, yet again suggesting their oligodendroglial nature. However, in some instances, it could not be clearly discerned whether these processes were astrocytic. Storage changes that combined CTB-like and banana-like profiles were further identified in endothelial cells (Figure 8E) of brain vessels. The endothelial storage, on a number of occasions, resulted in the formation of major intraluminal endothelial protrusions. To a lesser extent, storage bodies could also be detected in smooth muscle cells of the muscularis layer of larger-sized vessels (data not shown). 


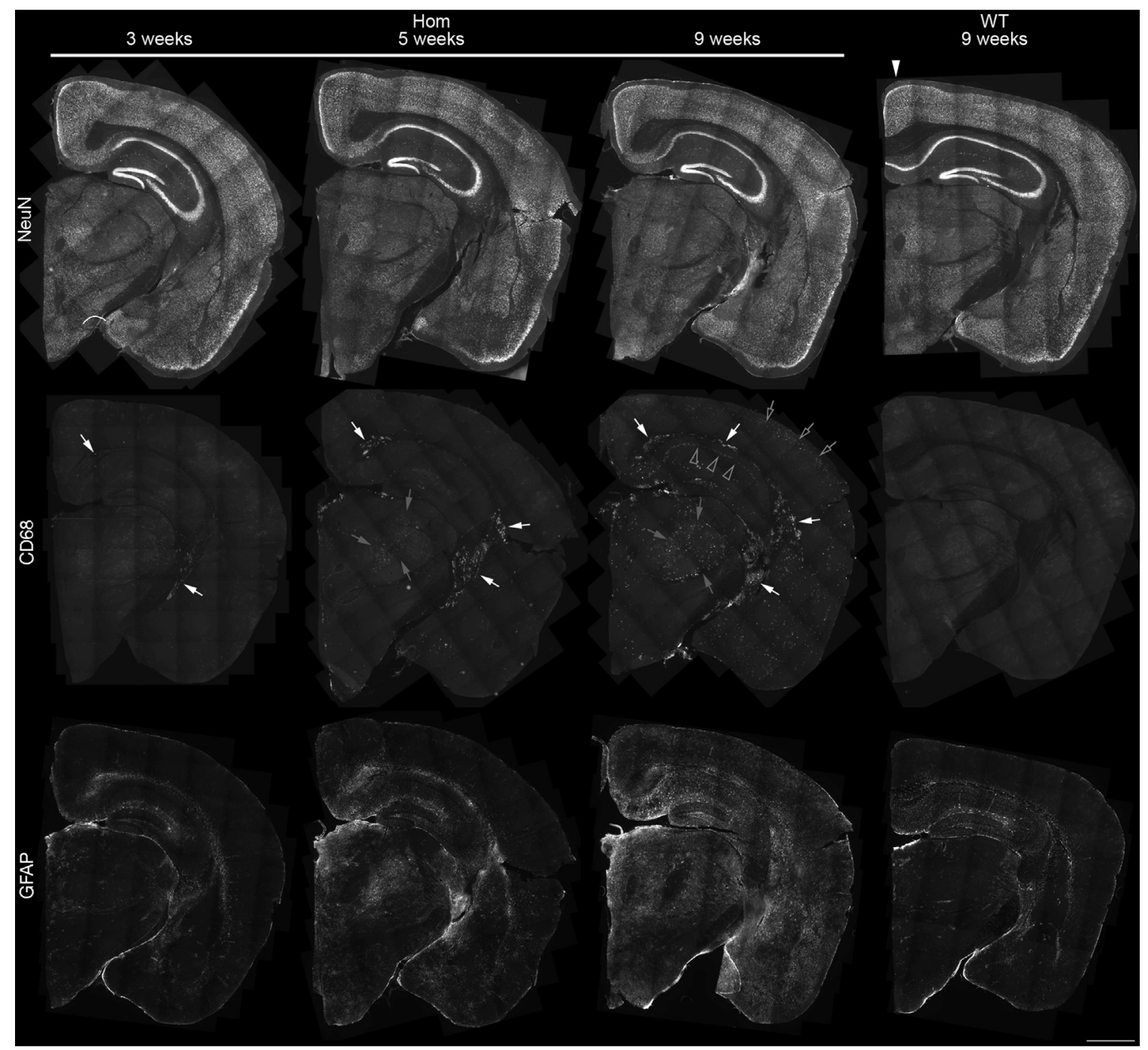

Figure 5 Temporal and spatial progress of the cell type-specific pathologic changes in the cerebrum of Asah1 $1^{\mathrm{P} 361 \mathrm{R} / \mathrm{P} 361 \mathrm{R}}$ homozygous (Hom) mice. Cerebral pathologic findings in 3-, 5-, and 9-week-old Hom mice compared with 9-week-old wild-type (WT) animals. Overall organization of the gray matter (GM) [highlighted by neuronal nuclei (NeuN)] is not substantially affected in Hom mice. CD68 ${ }^{+}$microglia and/or macrophages (white arrows) and glial fibrillary acidic protein (GFAP) astrocytic abnormalities are detectable already at 3 weeks of age in the external capsule (subcortical white matter), internal capsule, and hippocampal fimbria and are progressive with age in Hom animals. Likely discrete neurodegeneration in specific GM areas [thalamus (gray arrows), CA1 region of the hippocampus (gray hollow arrowheads), and layer II of the primary somatosensory (S1) cortex (gray hollow arrows)] is reflected by neuronophagic clustering of $\mathrm{CD}_{6} 8^{+}$microglia and/or macrophages. These pathologic changes first appear at 5 weeks and progress until 9 weeks of age. Pathologic changes of the external capsule (white arrows) are shown in detail in Figure 6, A and B. Hippocampal CA1 (gray hollow arrowheads) and layer II of S1 cortex (gray hollow arrows) changes are shown in detail in the Figure 6, C and D, respectively. White arrowhead highlights the approximate position of the sagittal section planes shown as mass spectrometry imaging data in Figure 3 . Scale bar $=1 \mathrm{~mm}$.

Contrary to the abnormal microglia and/or macrophages described earlier, the predominant finding in neurons were zebra body-like profiles (Figure 9A) that varied from finely striated (eg, PCs) (Figure 9B) to nearly cleared vesicles (eg, granule cell layer neurons) (Figure 9C) that did not form large confluent structures. Similar to histopathologic and CathD IHC findings (Supplemental Figure S4, A and B), EM did not reveal any major and/or widely distributed changes in overall neuronal cytoarchitecture (eg, cytoplasmic ballooning). In some brain areas where immunofluorescence analyses suggested neuronophagy (Figure 5), apoptoticlike changes (karyorrhexis) of NeuN could be identified (Figure 7B) in 9-week-old mice. We further searched for cytoplasmic non-membrane-bound protein aggregate-like structures; however, we identified only a minimum of such changes in neurons of Hom Farber mice.

Lastly, excess of CathD by IHC was found in choroid plexus cells already at 3 weeks of age (Figure 10A). Well-discernible and numerous CTBs and/or zebra-like storage profiles could be identified in choroid plexus cells in 

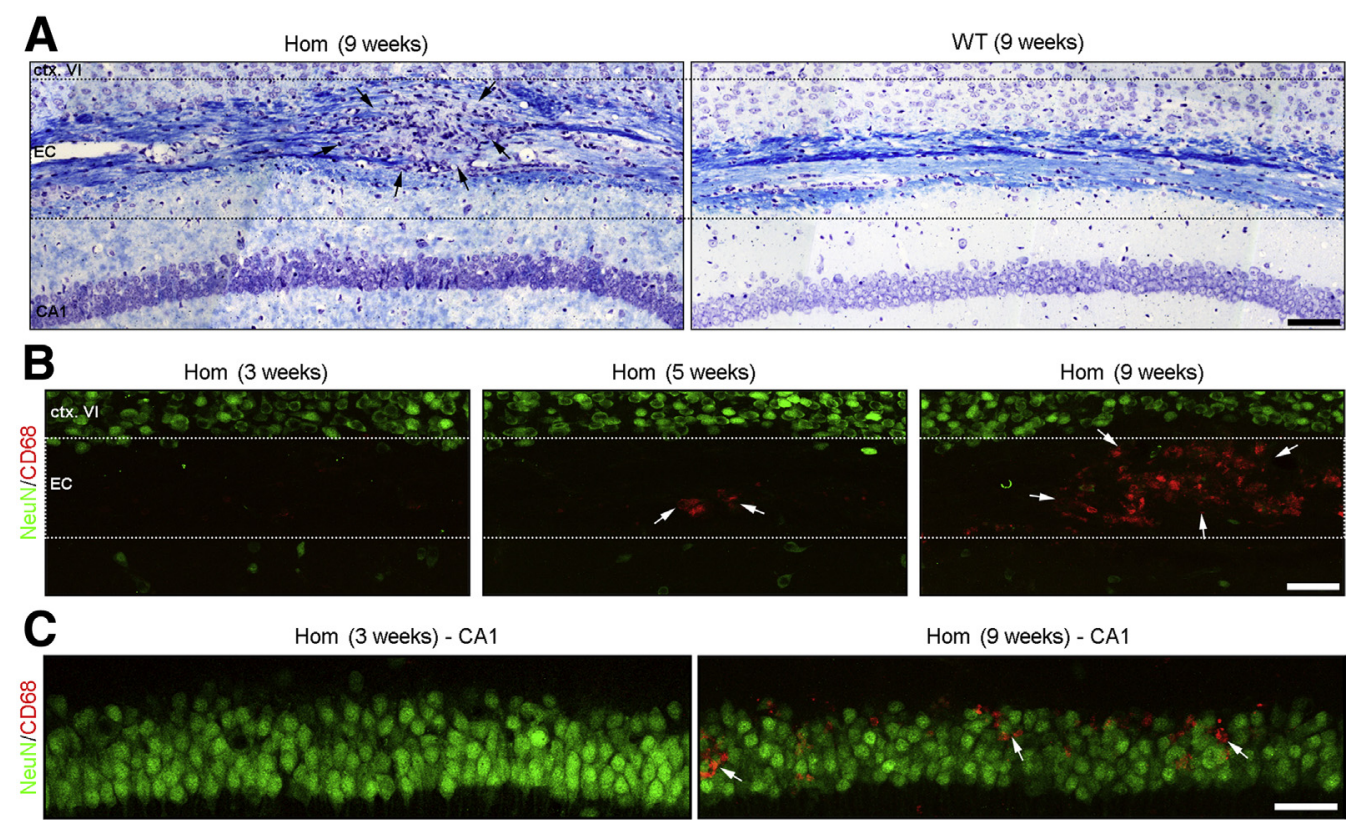

om (5 weeks)
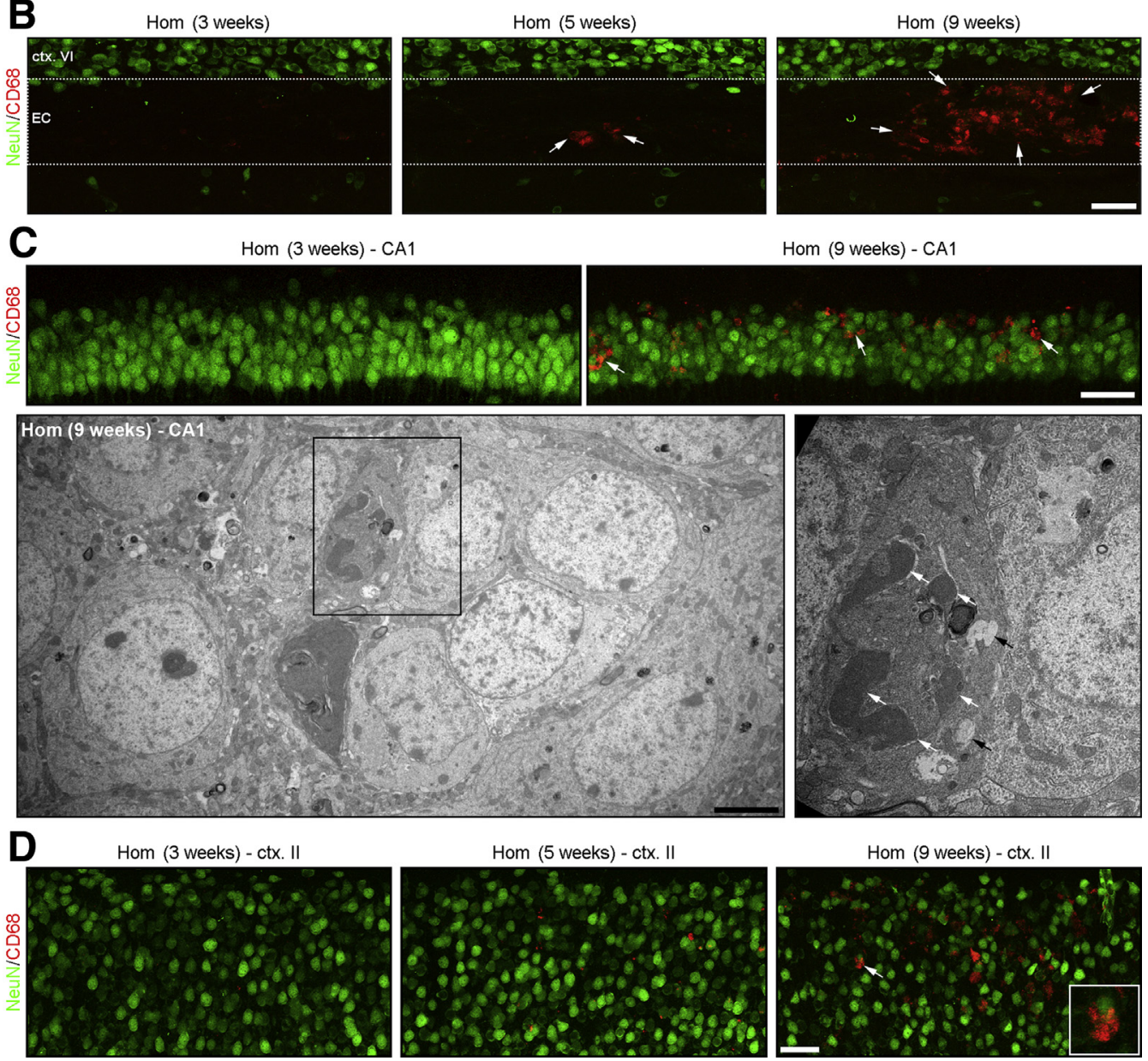

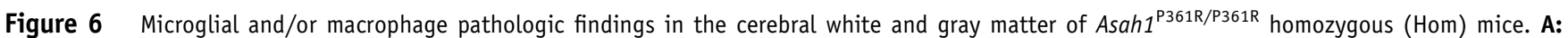
Luxol fast blue (LFB) staining outlines the external capsule (EC, subcortical white matter), which contains, in 9-week-old Hom animals, abnormally clustered cells (black arrows) that correspond to $\mathrm{CD}_{6} 8^{+}$microglia and/or macrophages (white arrows) in B. C: In CA1 hippocampal region neurodegeneration can be implied by localized microglial (CD68 ${ }^{+}$cells in red by co-immunofluorescence) neuronophagic clusters (white arrows) in 9 -week-old animals. Ultrastructural findings highlight individual degenerated neurons with karyorrhectic (blebbed) nuclei (white arrows) and cytoplasmic storage changes (black arrows) in CA1 hippocampal neurons at 9 weeks. D: Pathologic abnormalities similar to changes observed in CA1 can be identified also in the cortical layer II (here shown in the cortical somatosensory (S1) area). Inset (corresponds to the area highlighted by the white arrow) in the 9-weekold Hom animal shows a cluster of $\mathrm{CD}^{+} 8^{+}$(red) cells phagocytosing a neuronal nuclei (NeuN)-positive neuron (green). For neuroanatomic localization of areas shown in $\mathbf{A}-\mathbf{D}$ and also findings in 9-week-old wild-type animals, compare with Figure 5. Dotted lines in $\mathbf{A}$ and $\mathbf{B}$ highlight the EC (subcortical white matter). Immunofluorescence confocal image stacks (13- $\mu \mathrm{m}$ thick in $z)$ shown in B-D are presented as maximum intensity projections. Scale bars: $100 \mu \mathrm{m}(\mathbf{A}) ; 50 \mu \mathrm{m}(\mathbf{B}) ; 50 \mu \mathrm{m}$ (confocal) and $5 \mu \mathrm{m}$ (EM) (C); $50 \mu \mathrm{m}$ (D). CA1, CA1 region of hippocampus; ctx. II, cortical layer II; ctx. VI, cortical layer VI.

9-week-old Hom Farber mice (Figure 10B). On the contrary, ependymal cells seemed largely devoid of storage bodies or were affected only minimally. Similar to the histopathologic and immunofluorescence observations, we found local accumulation of storage laden microglia and/or macrophages in the subependymal zones (Figure 10C).

\section{Discussion}

FD is an ultrarare and severe multisystemic disorder. Given the small number of patients documented to date, the opportunity to study the clinical, biochemical, and cellular or tissue CNS-associated pathologic changes in FD has been 

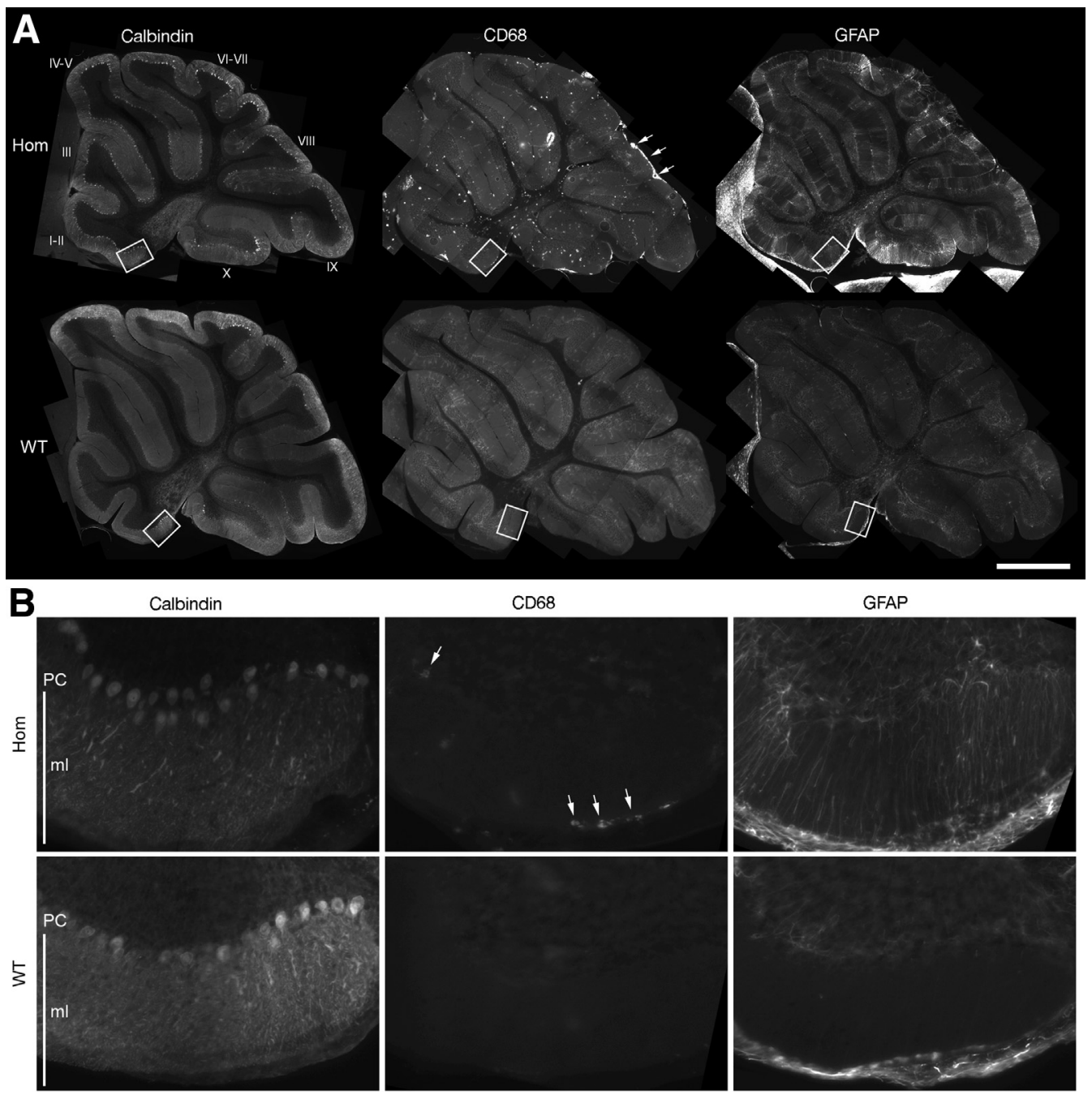

Figure 7 Cerebellar pathologic findings in Asah1 ${ }^{\mathrm{P} 361 \mathrm{R} / \mathrm{P} 361 \mathrm{R}}$ homozygous (Hom) mice. A: Comparison of 9-week-old Hom and wild-type (WT) cerebella does not suggest substantial and/or patterned degeneration of Purkinje cells (PCs). In Hom mice, lobules I to V and VIII, which are first affected by PC degeneration in murine models of several other lysosomal storage conditions, ${ }^{28-30}$ do not systematically lose PCs even at 9 weeks of age. The molecular layer (usually densely populated by reactive microglia when patterned PC degeneration develops) is devoid of changes indicative of clearance of PC dendritic arbors. In contrast, $\mathrm{CD} 8^{+}$microglia and/or macrophages are distributed in gray matter and white matter (WM) cerebellar areas. Local clustering of CD68 ${ }^{+}$cells can be observed within the PC layer, WM, and meninges (white arrows). Abnormal glial fibrillary acidic protein (GFAP) expression in the PC layer of Hom mice is compared with the normal patterns in WT mice. B: Details (correspond to boxed areas in A) of calbindin (PCs), CD68 (microglia and/or macrophages), and GFAP (astrocytes) immunofluorescent stains in the cerebellar lobule I. White arrows highlight $\mathrm{CD}^{+} 8^{+} \mathrm{microglia} / \mathrm{macrophages}$. Scale bar $=1 \mathrm{~mm}(\mathbf{A})$. $\mathrm{ml}$, molecular layer.

limited. However, several reports suggest neuronal lysosomal storage changes ${ }^{1,5,6}$ in individuals affected by FD. In contrast, data on lysosomal pathologic abnormalities involving nonneuronal CNS cell types is scant in FD. Individual brain MRI studies ${ }^{33}$ recently suggested loss of deep WM, hydrocephalus, and brain atrophy. Likewise, Cappellari et $\mathrm{al}^{34}$ documented increased size of pericerebellar sulci and the fourth ventricle in FD patient undergoing hematopoietic stem cell transplant.

We recently generated the first viable animal model of $\mathrm{FD},{ }^{13}$ and in this study we characterize the hallmark CNS deficits associated with ACDase deficiency. In addition to describing the key features of the brain disease, we also sought to identify readouts that could be used to help gauge the effect of novel treatment schemes for FD.

As noted earlier, Asahl $1^{\mathrm{P} 361 \mathrm{R} / \mathrm{P} 361 \mathrm{R}}$ (Hom Farber) mice present with a progressive and lethal phenotype. Macrophages are affected by ACDase deficiency and are thought to be key contributors to the progression of a wide range of tissue pathologic findings observed in Hom Farber mice. Indeed, there is an increase in circulating monocytes, and abnormal macrophages are numerous in the skin and liver. Disease-specific macrophages were previously also found to disrupt neuronal tracts in the spinal cord and to push on axons and affect myelin sheaths in sciatic nerves. ${ }^{13}$ These cells also infiltrate bone marrow, thymus, spleen, 

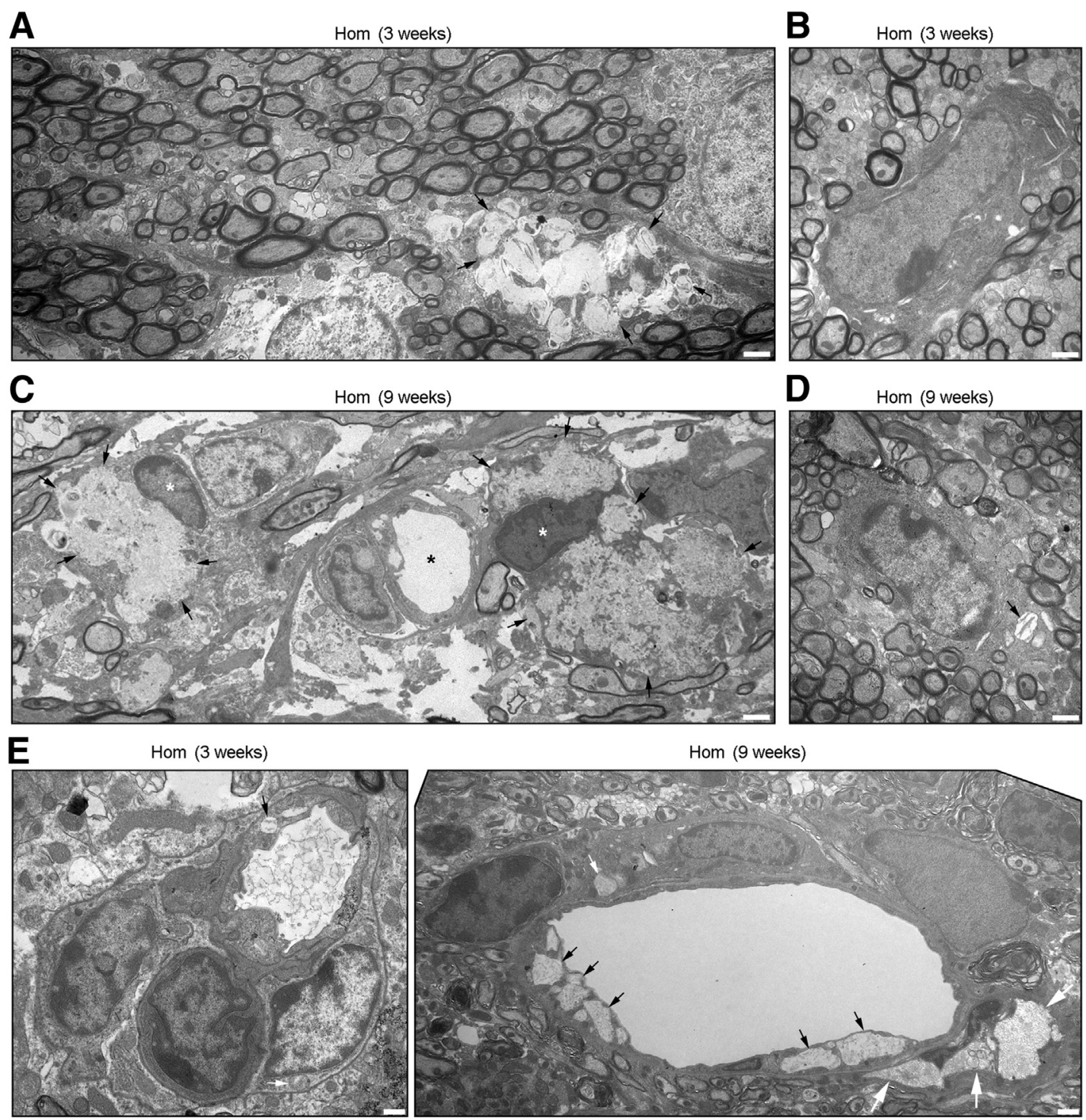

Figure 8 White matter (WM) and vascular ultrastructural storage pathologic findings in Asah1 $1^{\mathrm{P} 361 \mathrm{R} / \mathrm{P} 361 \mathrm{R}}$ homozygous (Hom) mice. A: Electron microscopy reveals storage changes in microglia and/or macrophages in subcortical WM already at 3 weeks of age (black arrows). B: Cells with oligodendroglial morphologic characteristics in Hom animals are largely devoid of storage vacuoles at 3 weeks. C: At 9 weeks of age, the storage bodies (black arrows) in microglial and/or macrophage-like cells are confluent. These abnormal cells (nuclei highlighted by white asterisks) form granulomatous clusters (compare with Figure 5 and Figure 6, A and B), many of which are located close to vessels (the vascular lumen is highlighted by the black asterisk). D: Cytoplasmic zebra-like storage changes (black arrow) can be identified in WM cells with oligodendroglial ultrastructural characteristics in 9-week-old Hom animals. E: Storage in endothelial (black arrows) and perivascular macrophages (small white arrow) is present in Hom brain vessels (cerebellar WM vessels are shown) already at 3 weeks of age. At 9 weeks, endothelial (black arrows), pericytic (large white arrows), and perivascular macrophage (small white arrow) storage pathologic changes are extensively developed. Scale bar $=1 \mu \mathrm{m}(\mathbf{A}-\mathbf{E})$.

and lymph nodes ${ }^{13}$ and contribute to the overall perturbed hematopoiesis we previously described in Hom Farber mice. ${ }^{14}$

Given their severely impaired activity and motor coordination, a limited number of behavioral tests were chosen for Hom Farber mice. Similar limitations apply to testing cognitive abilities of these mice. Furthermore, the behavioral phenotype that we describe in this study in Hom Farber mice should be interpreted within the context of their short lifespan and the progressive nature of their peripheral organ pathologic features. As a result, it is relatively difficult to establish clear neuroanatomical and neuropathologic correlates for individual behavioral abnormalities observed in these mice. As a dominant feature, Hom Farber mice become increasingly weaker over time compared with WT mice. It is likely that the weak grip strength and compromised locomotion and motor coordination observed in Hom Farber mice cannot be attributed solely to the pathologic changes in the cerebral and cerebellar motor control centers. Aside from the systemic toxic effects of the visceral disease, 

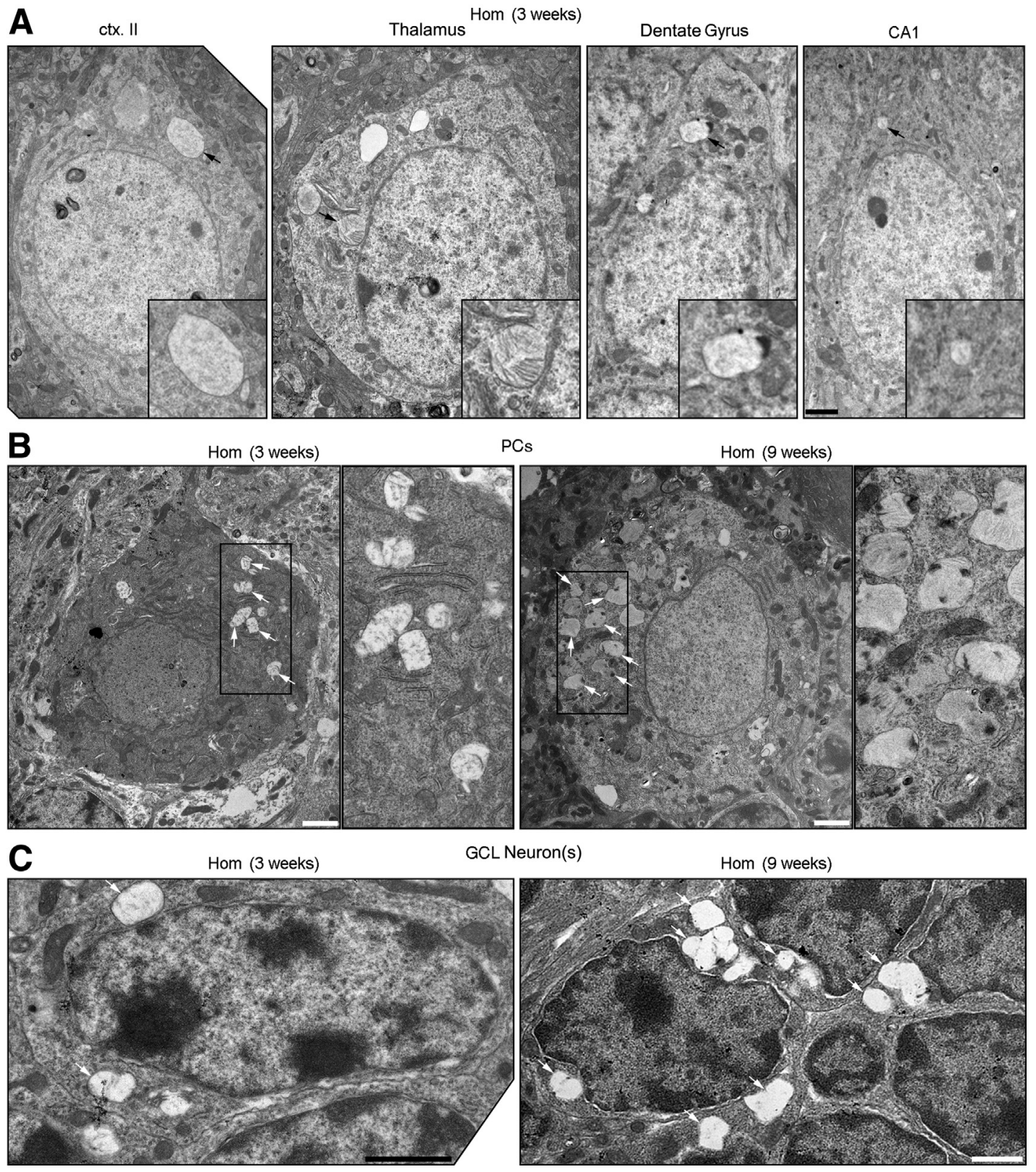

Figure 9 Neuronal ultrastructural storage pathologic findings in Asah1 ${ }^{\mathrm{P} 361 \mathrm{R} / \mathrm{P} 361 \mathrm{R}}$ homozygous (Hom) mice. A: Neuronal storage changes (shown in neurons of various regions) are detectable already at 3 weeks of age. Storage bodies (highlighted by black arrows and shown enlarged in the insets) often present as zebra body-like structures. B: Progressive storage changes (white arrows) are shown in Purkinje cells (PCs) from anterior cerebellar zones (lobules I to V). Zebra-like storage bodies have variable appearance and only occasionally form larger confluent structures. C: Storage bodies (white arrows) in granule cell layer (GCL) have cleared appearance. CA1, CA1 region of hippocampus; ctx. II, cortical layer II. Scale bar $=1 \mu \mathrm{m}(\mathbf{A}-\mathbf{C})$.

additional contributors to the muscle weakness could be (still unidentified) degeneration or dysfunction of spinal motor neurons (noticed by Moser et $\mathrm{al}^{6}$ in the human FD), pathologic changes in peripheral motor nerves, and theoretically also improper communication at the neuromuscular junction and/or myopathy.

Although behavioral studies do not pinpoint specific regions of brain that contribute to the clinical phenotype, our biochemical and morphologic studies identify a broad range of abnormalities at the subcellular, cellular, and organ levels. At the subcellular level, a plethora of neuronal and nonneuronal cell types develop storage changes compatible with those previously identified in nonneuronal tissues of FD patients ${ }^{2-4}$ and Hom Farber mice. Regardless of the complexity, the cerebral pathologic findings in Hom Farber mice can be categorized into two major areas based on the cell type affected: $\mathrm{CD}^{+} 8^{+}$microglia and/or macrophages and neurons. Similar to nonneuronal tissues, the CNS in the Farber mice is extensively affected by the granuloma-like accumulation of the abnormal $\mathrm{CD} 68^{+}$cells. In the cerebrum, this abnormality is initiated and progresses in the WM areas, often associating with cerebral vessels as 

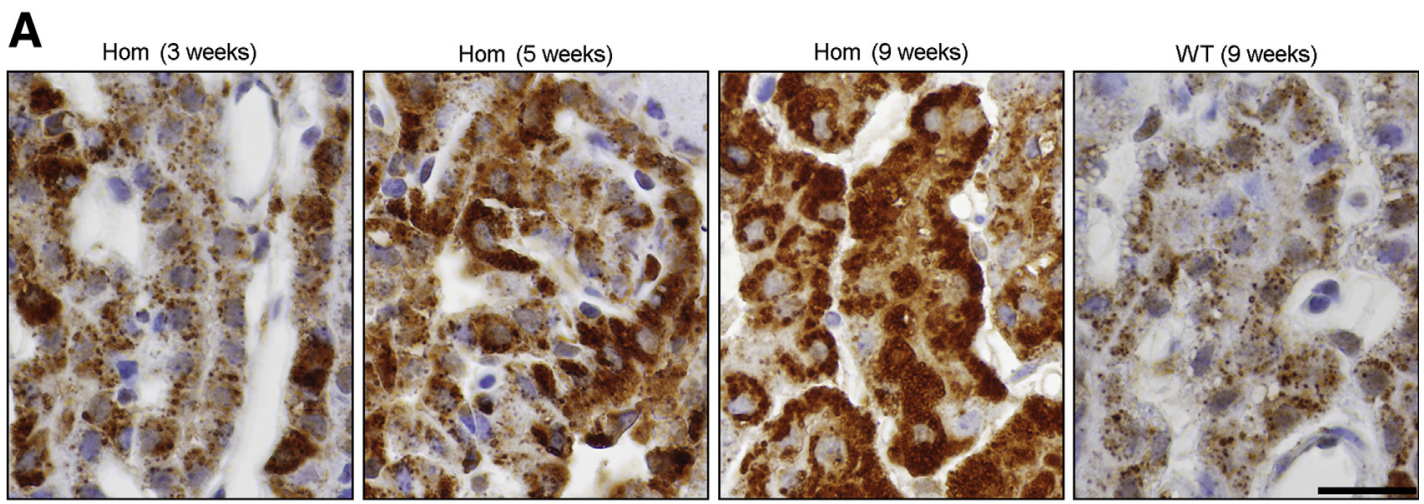

B
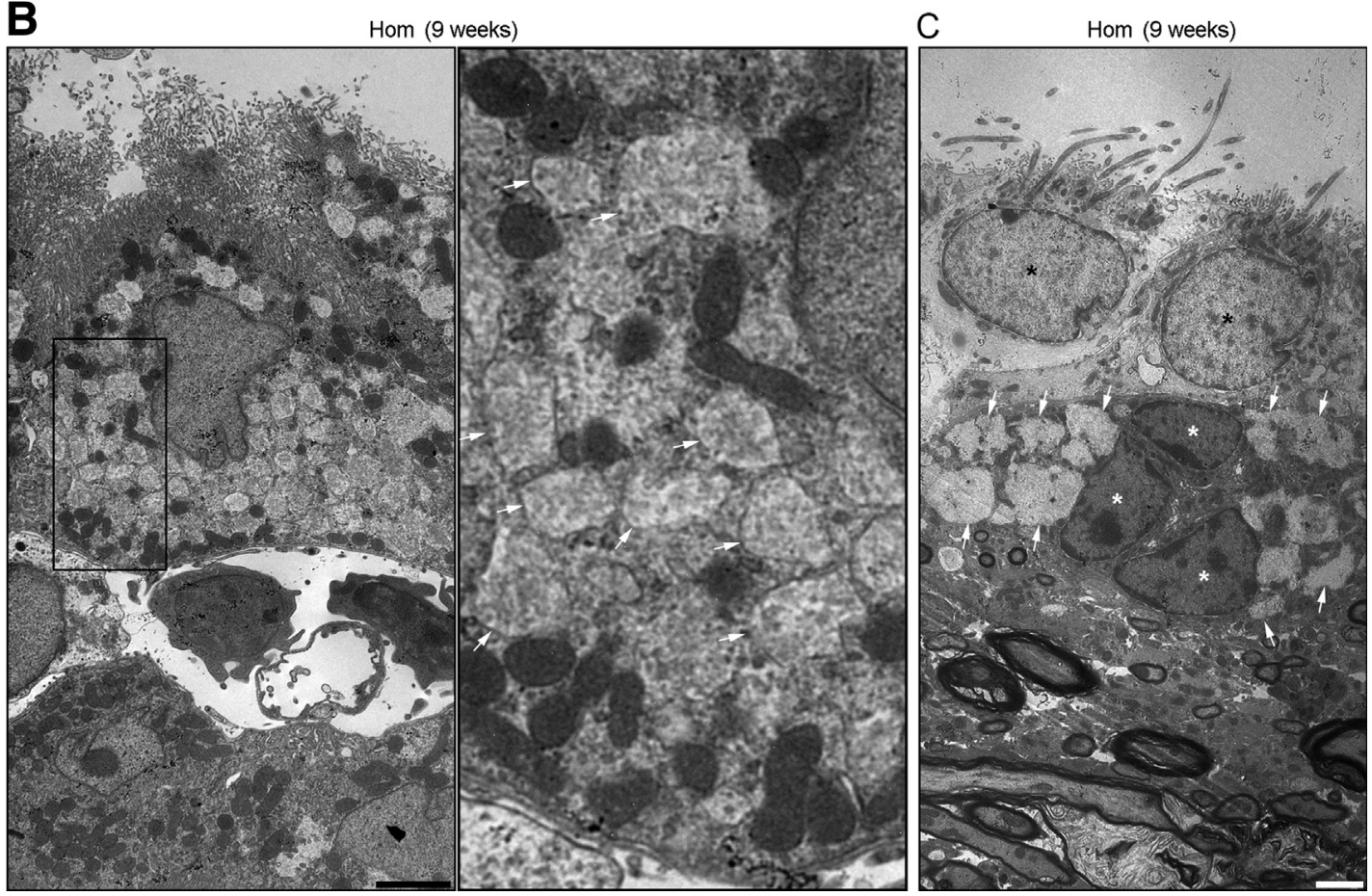

Figure 10 Choroid plexus and ependymal storage pathologic findings in Asah1 $1^{\mathrm{P} 361 \mathrm{R} / \mathrm{P} 361 \mathrm{R}}$ homozygous (Hom) mice. A: Accumulation of cathepsin D-positive (lysosomal luminal marker) vacuoles in choroid plexus (lateral ventricles are shown) cells is initiated already in 3-week-old Hom mice and progresses with age. B: Choroid plexus cells (fourth ventricle) in Hom animals contain curvilinear tubular bodies and zebra-like storage bodies (white arrows) by electron microscopy. C: Ependymal cells (fourth ventricle, nuclei marked by black asterisks) seem to be relatively spared of storage changes; nonetheless, subependymal zone contains microglia and/or macrophages (nuclei marked by white asterisks) filled with confluent storage bodies (white arrows). Scale bars: $20 \mu \mathrm{m}(\mathbf{A}) ; 1 \mu \mathrm{m}(\mathbf{B}$ and $\mathbf{C})$.

perivascular cuffs. At later stages of disease, it is likely that a fraction of these clustered abnormal cells degenerate and disintegrate and release their content into the extracellular space. Given this vessel-linked distribution pattern in the $\mathrm{WM}$, it is possible that these abnormal cells do not represent an endogenous brain pool of microglia and/or macrophages but rather originate from the periphery in a manner similar to other tissues in Hom Farber mice.

Various cerebral neuronal populations are also affected by the subcellular storage changes. Interestingly, the overall morphologic alteration of neurons is not as pronounced as in the $\mathrm{CD}_{68}{ }^{+}$microglia and/or macrophages. However, specific neuronal populations seem to be more sensitive to the primary enzymatic defect than others and degenerate as a result.
This localized degeneration is associated with clearance by microglia that differ morphologically from the granulomalike accumulations of foamy $\mathrm{CD}^{+} 8^{+}$cells in WM areas. Interestingly, some of the neuroanatomic areas likely affected by neuronal degeneration (eg, specific thalamic nuclei) were previously reported as similarly compromised in mouse models of other lysosomal storage diseases. ${ }^{31,32}$ Also important for future detailed studies will be to identify whether particular functionally specialized neuronal populations (eg, GABAergic interneurons), previously found to be preferentially compromised in lysosomal storage conditions, ${ }^{35}$ are also affected in Hom Farber mice.

As an additional pathologic feature in brains of Hom Farber mice, we found ultrastructural storage 
changes also in the WM cells with oligodendroglial (and potentially also astrocytic) morphologic characteristics; however, the extent of the effect these changes have on myelin build-up and overall WM integrity requires further detailed studies.

The dominant neuronal findings in the cerebellum of Hom Farber mice are the accumulation of zebra bodies in PCs and excess of the cleared storage profiles in granule cell layer neurons. Similar to the cerebrum, the local accumulation of abnormal microglia and/or macrophages could be identified in cerebellar WM and GM. Cerebellar WM oligodendroglia-like cells also presented with ultrastructural storage profiles similar to those found in cerebrum. Critically, however, in Hom Farber mice, we did not observe the patterned degeneration of PCs and/or the microglial and/or macrophage and astrocytic pathologic changes usually associating with this degeneration in mouse models of other lysosomal storage diseases. ${ }^{28-30}$

Findings in the cerebral vasculature are also an important part of the overall CNS pathologic features in Hom Farber mice. Endothelial cells contained large amounts of storage material that organized into large confluent storage bodies. By forming luminal protrusions, these bodies on many occasions substantially disfigured the overall morphologic features of the endothelial cells. Given the size of these protrusions and the global extent of this pathologic feature, it is plausible that the subcellular storage pathologic features might affect global blood perfusion of the brain and negatively contribute to the neurologic phenotype in this disease. As outlined above, the extensive perivascular microglial and/or macrophage pathologic accumulation associated with vessels in both the cerebrum and cerebellum could also potentially contribute to impaired perfusion.

As reported before, ${ }^{13}$ hydrocephalus that affects all ventricles was identified in a fraction of Hom Farber mouse brains. The general causes of hydrocephalus include increased production, compromised resorption, or obstructed flow of the cerebrospinal fluid (CSF). Alternatively, the volume of CSF increases with the atrophy of the brain tissue, which is not likely in Hom Farber mice given their lifespan and lack of evidence of numerically large-scale neuronal degeneration. Reviewing abnormalities in cell types participating in CSF homeostasis and circulation in Hom Farber mice, we identified subcellular storage pathologic changes in choroid plexus cells. Furthermore, abnormal $\mathrm{CD}^{+} 8^{+}$cells were present in the stroma of choroid plexi. Importantly, the storage abnormalities seemed minimal in ependymal cells. However, local granulomatous accumulations of the abnormal microglia and/or macrophages could be found in the subependymal periventricular areas and in the meninges. The latter localization could possibly also include local negative effects of these abnormal cells in the arachnoid granulations, which are sites critical for CSF resorption. Alternatively, we could speculate that the subcellular pathologic alterations in choroid plexus cells may trigger CSF overproduction. Overall, however, the exact mechanisms causing the communicating hydrocephalus in Hom Farber mice still remain unresolved.

Although comparisons are difficult to establish because the human FD neuropathologic studies are sporadic, Moser et $\mathrm{al}^{6}$ reported neuronal storage changes, spatially restricted cortical and subcortical neurodegeneration associated with neuronophagy, fibrous gliosis, microglial proliferation, and some myelin loss in a 9.5-month-old patient with FD. Interestingly, cortical layers 2 to 3 were preferentially affected by neurodegeneration, and neurodegeneration could also be found in the hippocampus of this FD patient. Furthermore, similar to our findings in Hom Farber mice, a normal complement of PCs, some of which appeared distended, was reported by Moser et al. ${ }^{6}$ Interestingly, the latter FD case report mentions microglial and macrophage pathologic changes only briefly.

Similarly, little information on the lipid storage in the brain of FD patients is available. Accumulation of Cer in the visceral organs and cerebral WM of severely affected FD patients was first reported in $1967^{36}$ and $1969 .^{6}$ In the cerebellum, storage of hydroxy-C24:0 and C22:0 Cer was documented by Sugita et al. ${ }^{37,38}$ Compared with control individuals, the non-hydroxy fatty acid-containing Cer content was doubled in the cerebellum of the FD patient, whereas that of the 2-hydroxy fatty acid-containing Cer was increased by almost 50-fold, representing more than one-third of total Cers, a finding similar to that reported in Figure 2. Accumulation of a glycolipid in brain was also observed $^{6,39}$ in FD patients.

As a class of bioactive-signaling molecules, it is important to know which specific Cer species are accumulating in FD because they are likely the initiating agents of the observed downstream histopathologic and behavioral changes reported here. However, current knowledge of the functions of individual sphingolipid species is limited. Most studies have been centered on the role of Cers in apoptosis and autophagy, including in neurons. ${ }^{40}$ C16:0-Cer is involved at multiple points of the apoptotic signaling pathway (reviewed by Grösch et $\mathrm{al}^{41}$ ), whereas C16:0-dhCer is elevated in response to molecules that induce autophagy. ${ }^{42,43}$ Both C16:0-Cer and C16:0dhCer levels had large fold elevations in brains from Hom Farber mice, and some changes indicative of apoptosis were seen in neurons. Increases in the C16-Cers and decreases in the C18-Cers are implicated in cerebellar PC neurodegeneration in the flincher mouse, ${ }^{44}$ yet in the Hom Farber mice we did not see significant PC loss, perhaps because of an elevation instead of a reduction of $\mathrm{C} 18$.

All such attempts to link particular Cer species with biological function not only require knowledge on the cell types in which they accumulate but also the subcellular location(s). It could be argued that the accumulation of Cers 
in FD, given the loss of function of ACDase, would be limited to the lumen of late endosomes and lysosomes. In such a case, little effect on cell death pathways might be expected; rather, deficiency of Cer precursors for various metabolic pathways might be more likely. Unfortunately, detailed information on subcellular location and dynamics of turnover remains largely unavailable at this time. Thus, in the FD mouse model, cells may be compensating for the entrapment of Cer precursors secondary to ACDase deficiency through changes in the synthesis of Cers (such as by altered $C e r S$ ) and/or by increased degradation by other ceramidases (eg, neutral ceramidase). The results of the sphingoid base analyses support this hypothesis. Sph is the direct product of ACDase activity, so with dysfunctional ACDase there should be less Sph. Instead, Sph was increased, whereas other sphingoid bases remain unchanged. These results suggest that neutral and/or alkaline ceramidases may compensate somewhat for the ACDase deficiency. This compensation appears to be partially effective because the brain has a relatively low degree of Cer accumulation compared with other organs (the spleen, liver, lung, heart and kidney). ${ }^{13}$ However, there are still Cer species that are altered, suggesting that neutral and alkaline ceramidases have specificity for particular acyl chains. Interestingly, the Cer species exhibiting the lowest fold elevation in Hom Farber mice is C18:1-Cer, a species that is known to be preferentially catabolized by the alkaline ceramidase ACER3. ${ }^{45}$

Ceramide changes have been seen in several other neurologic diseases. Elevated levels of C16, C18, C20, and C24 Cers have been reported in the brains of patients with Alzheimer disease and other neurodegenerative diseases. ${ }^{46,47}$ Astrocytes surrounding Alzheimer disease plaques mostly have $\mathrm{C} 18$ and C24:1 Cers. ${ }^{48}$ In fact, Cers have been reported to induce generation of the pathologic molecule, $\mathrm{A} \beta .^{49}$ In Parkinson disease, there is a general Cer decrease in the anterior cingulate cortex. ${ }^{50}$ Decreased C18-Cers also lead to progressive myoclonus epilepsy. ${ }^{51}$ These latter changes are accompanied by alterations in the expression of CerS genes, which encode for Cer synthases. Injection of Cers into the brains of rat pups alters their behavioral phenotype and lipid composition similar to findings in the Hom Farber mice. ${ }^{52}$ However, as described above, lack of detailed information on where the Cers are located subcellularly limit insights into a role in pathogenesis.

In addition to the absolute value of the accumulation of Cers, the relative abundance of Cer species is also important for normal cell, tissue, and organ function. In other neurologic disorders, such as Alzheimer disease, the fractional composition of each species is unchanged between normal and diseased brains. ${ }^{46}$ In this study, we saw changes in the fractional composition. In brains from Hom Farber mice, Cer-OH species had the largest fold-increase over WT mice. This finding may be attributed to the high abundance of hydroxylated galactosphingolipids in myelin; thus, turnover of these sphingolipids leads to an accumulation of Cer-OHs. The increased levels of MHC-OH relative to MHC would then likely represent hydroxylated galactosylceramide. This finding, however, suggests that galactosylceramide turnover is much greater than that for other sphingolipids or that sphingolipids in myelin are more rapidly turned over. Elevation of DHC levels has also been seen in other sphingolipidoses. This increase (with little change in glucosylceramide and galactosylceramide), as well as that of GM3 ganglioside, could be in line with the traffic jam described to accompany many lysosomal lipid storage disorders. ${ }^{53}$ We also used MALDI-MSI to identify specific locations of a subset of Cers and gangliosides. Further analysis of this sort examining the entire brain may help in connecting the sphingolipid accumulation to brain regions affected, and link this to behavioral outcomes. For example, the cerebellum plays a role in controlling motor coordination, and the C16:0-, C18:0-, and C20:0-Cers are all elevated in that region. These factors may be contributing to the motor deficits seen in the behavioral tests. Because the FD model will be used for testing of new genetic therapeutic approaches, the ability to monitor the sites of accumulation after therapeutic intervention will provide crucial tissue-specific outcome measures.

\section{Conclusions}

Neurologic disease represents a critical part of the clinical phenotype of FD. However, given the lack of patient tissues and previous long-term unavailability of a suitable animal model, the CNS pathologic abnormalities have, so far, remained largely unexplored in FD. Using our recently developed mouse model, we found the rapidly progressing and profound biochemical and structural effects of the ACDase deficiency on a broad array of neuronal and nonneuronal cell types and the brain as a whole. By characterizing these hallmark abnormalities, we have not only demonstrated the crucial physiologic roles of the lysosomal ACDase-mediated sphingolipid degradation for CNS integrity but also outlined the range of target cell types and pathologic changes for any future therapies of this devastating and currently untreatable neurovisceral disease.

\section{Acknowledgments}

We thank Stéphane Carpentier for brain lipid extractions; Min Hui (MRI facility-Albert Einstein College of Medicine, New York City, NY), Jennifer Leddy, and Gloria Stephney for tissue processing for light and electron microscopy (Albert Einstein College of Medicine, New York City, NY), and Marie Kolarova for immunohistochemistry (Institute of Inherited Metabolic Disorders, Charles University, 1st Faculty of Medicine). 


\section{Supplemental Data}

Supplemental material for this article can be found at http://dx.doi.org/10.1016/j.ajpath.2016.12.005.

\section{References}

1. Levade T, Sandhoff K, Schulze H, Medin JA: Acid ceramidase deficiency: Farber lipogranulomatosis. Edited by Valle D, Vogelstein B, Kinzler KW, Antonarakis SE, Ballabio A, Mitchell G. In The Online Metabolic and Molecular Bases of Inherited Diseases. New York: McGraw-Hill, 2009

2. Abenoza P, Sibley RK: Farber's disease: a fine structural study. Ultrastruct Pathol 1987, 11:397-403

3. Burck U, Moser HW, Goebel HH, Gruttner R, Held KR: A case of lipogranulomatosis Farber: some clinical and ultrastructural aspects. Eur J Pediatr 1985, 143:203-208

4. Zappatini-Tommasi L, Dumontel C, Guibaud P, Girod C: Farber disease: an ultrastructural study: report of a case and review of the literature. Virchows Arch A Pathol Anat Histopathol 1992, 420: $281-290$

5. Molz G: Farber's disease: pathologic anatomical findings [in French]. Virchows Arch A Pathol Pathol Anat 1968, 344:86-99

6. Moser HW, Prensky AL, Wolfe HJ, Rosman NP: Farber's lipogranulomatosis: report of a case and demonstration of an excess of free ceramide and ganglioside. Am J Med 1969, 47:869-890

7. Zhou J, Tawk M, Tiziano FD, Veillet J, Bayes M, Nolent F, Garcia V, Servidei S, Bertini E, Castro-Giner F, Renda Y, Carpentier S, Andrieu-Abadie N, Gut I, Levade T, Topaloglu H, Melki J: Spinal muscular atrophy associated with progressive myoclonic epilepsy is caused by mutations in ASAH1. Am J Hum Genet 2012, 91:5-14

8. Dyment DA, Sell E, Vanstone MR, Smith AC, Garandeau D, Garcia V, Carpentier S, Le Trionnaire E, Sabourdy F, Beaulieu CL, Schwartzentruber JA, McMillan HJ; FORGE Canada Consortium, Majewski J, Bulman DE, Levade T, Boycott KM: Evidence for clinical, genetic and biochemical variability in spinal muscular atrophy with progressive myoclonic epilepsy. Clin Genet 2014, 86: $558-563$

9. Gan JJ, Garcia V, Tian J, Tagliati M, Parisi JE, Chung JM, Lewis R, Baloh R, Levade T, Pierson TM: Acid ceramidase deficiency associated with spinal muscular atrophy with progressive myoclonic epilepsy. Neuromuscul Disord 2015, 25:959-963

10. Giraldez BG, Guerrero-Lopez R, Ortega-Moreno L, Verdu A, Carrascosa-Romero MC, Garcia-Campos O, Garcia-Munozguren S, Pardal-Fernandez JM, Serratosa JM: Uniparental disomy as a cause of spinal muscular atrophy and progressive myoclonic epilepsy: phenotypic homogeneity due to the homozygous c.125C $>$ T mutation in ASAH1. Neuromuscul Disord 2015, 25:222-224

11. Rubboli G, Veggiotti P, Pini A, Berardinelli A, Cantalupo G, Bertini E, Tiziano FD, D’Amico A, Piazza E, Abiusi E, Fiori S, Pasini E, Darra F, Gobbi G, Michelucci R: Spinal muscular atrophy associated with progressive myoclonic epilepsy: a rare condition caused by mutations in ASAH1. Epilepsia 2015, 56:692-698

12. Li CM, Park JH, He X, Levy B, Chen F, Arai K, Adler DA, Disteche CM, Koch J, Sandhoff K, Schuchman EH: The human acid ceramidase gene (ASAH): structure, chromosomal location, mutation analysis, and expression. Genomics 1999, 62:223-231

13. Alayoubi AM, Wang JC, Au BC, Carpentier S, Garcia V, Dworski S, El-Ghamrasni S, Kirouac KN, Exertier MJ, Xiong ZJ, Prive GG, Simonaro CM, Casas J, Fabrias G, Schuchman EH, Turner PV, Hakem R, Levade T, Medin JA: Systemic ceramide accumulation leads to severe and varied pathological consequences. EMBO Mol Med 2013, 5:827-842

14. Dworski S, Berger A, Furlonger C, Moreau JM, Yoshimitsu M, Trentadue J, Au BC, Paige CJ, Medin JA: Markedly perturbed hematopoiesis in acid ceramidase deficient mice. Haematologica 2015, 100:e162-e165

15. Martin P, Patrick P, Bateson G, Bateson P: Measuring Behaviour: An Introductory Guide. Cambridge, Cambridge University Press, 1993

16. Thomas A, Burant A, Bui N, Graham D, Yuva-Paylor LA, Paylor R: Marble burying reflects a repetitive and perseverative behavior more than novelty-induced anxiety. Psychopharmacology (Berl) 2009, 204: 361-373

17. Carlson CG, Rutter J, Bledsoe C, Singh R, Hoff H, Bruemmer K, Sesti J, Gatti F, Berge J, McCarthy L: A simple protocol for assessing inter-trial and inter-examiner reliability for two noninvasive measures of limb muscle strength. J Neurosci Methods 2010, 186:226-230

18. Bedia C, Casas J, Andrieu-Abadie N, Fabrias G, Levade T: Acid ceramidase expression modulates the sensitivity of A375 melanoma cells to dacarbazine. J Biol Chem 2011, 286:28200-28209

19. Garanto A, Mandal NA, Egido-Gabas M, Marfany G, Fabrias G, Anderson RE, Casas J, Gonzalez-Duarte R: Specific sphingolipid content decrease in Cerkl knockdown mouse retinas. Exp Eye Res 2013, 110:96-106

20. Franklin KBJ, Paxinos G: The Mouse Brain in Stereotaxic Coordinates. ed 3. New York, Elsevier, Inc., 2008

21. Jones EE, Dworski S, Canals D, Casas J, Fabrias G, Schoenling D, Levade T, Denlinger C, Hannun YA, Medin JA, Drake RR: On-tissue localization of ceramides and other sphingolipids by MALDI mass spectrometry imaging. Anal Chem 2014, 86:8303-8311

22. McGlynn R, Dobrenis K, Walkley SU: Differential subcellular localization of cholesterol, gangliosides, and glycosaminoglycans in murine models of mucopolysaccharide storage disorders. J Comp Neurol 2004, 480:415-426

23. Sikora J, Leddy J, Gulinello M, Walkley SU: X-linked Christianson syndrome: heterozygous female Slc9a6 knockout mice develop mosaic neuropathological changes and related behavioral abnormalities. Dis Model Mech 2016, 9:13-23

24. Micsenyi MC, Sikora J, Stephney G, Dobrenis K, Walkley SU: Lysosomal membrane permeability stimulates protein aggregate formation in neurons of a lysosomal disease. J Neurosci 2013, 33: 10815-10827

25. Peters A, Palay SL, Webster HD: Fine Structure of the Nervous System: Neurons and Their Supporting Cells. ed 3. New York, Oxford University Press, 1991

26. Fletcher JL, Kondagari GS, Vite CH, Williamson P, Taylor RM: Oligodendrocyte loss during the disease course in a canine model of the lysosomal storage disease fucosidosis. J Neuropathol Exp Neurol 2014, 73:536-547

27. Guillemin GJ, Brew BJ: Microglia, macrophages, perivascular macrophages, and pericytes: a review of function and identification. J Leukoc Biol 2004, 75:388-397

28. Macauley SL, Sidman RL, Schuchman EH, Taksir T, Stewart GR: Neuropathology of the acid sphingomyelinase knockout mouse model of Niemann-Pick A disease including structure-function studies associated with cerebellar Purkinje cell degeneration. Exp Neurol 2008, 214:181-192

29. Praggastis M, Tortelli B, Zhang J, Fujiwara H, Sidhu R, Chacko A, Chen Z, Chung C, Lieberman AP, Sikora J, Davidson C, Walkley SU, Pipalia NH, Maxfield FR, Schaffer JE, Ory DS: A murine Niemann-Pick C1 I1061T knock-in model recapitulates the pathological features of the most prevalent human disease allele. J Neurosci 2015, 35:8091-8106

30. Sarna JR, Larouche M, Marzban H, Sillitoe RV, Rancourt DE, Hawkes R: Patterned Purkinje cell degeneration in mouse model of Niemann-Pick type C disease. J Comp Neurol 2003, 456: 279-291

31. Partanen S, Haapanen A, Kielar C, Pontikis C, Alexander N, Inkinen T, Saftig P, Gillingwater TH, Cooper JD, Tyynela J: Synaptic changes in the thalamocortical system of cathepsin D-deficient mice: a model of human congenital neuronal ceroid-lipofuscinosis. J Neuropathol Exp Neurol 2008, 67:16-29 
32. Pressey SN, Smith DA, Wong AM, Platt FM, Cooper JD: Early glial activation, synaptic changes and axonal pathology in the thalamocortical system of Niemann-Pick type C1 mice. Neurobiol Dis 2012, 45:1086-1100

33. Chedrawi AK, Al-Hassnan ZN, Al-Muhaizea M, Colak D, Al-Younes B, Albakheet A, Tulba S, Kaya N: Novel V97G ASAH1 mutation found in Farber disease patients: unique appearance of the disease with an intermediate severity, and marked early involvement of central and peripheral nervous system. Brain Dev 2012, 34: 400-404

34. Cappellari AM, Torcoletti M, Triulzi F, Corona F: Nervous system involvement in Farber disease. J Inherit Metab Dis 2016, 39:149-150

35. Walkley SU, Baker HJ, Rattazzi MC, Haskins ME, Wu JY: Neuroaxonal dystrophy in neuronal storage disorders: evidence for major GABAergic neuron involvement. J Neurol Sci 1991, 104:1-8

36. Prensky A, Ferreira G, Carr S, Moser H: Ceramide and ganglioside accumulation in Farber's lipogranulomatosis. Proc Soc Exp Biol Med $1967,126: 725-728$

37. Sugita M, Iwamori M, Evans J, McCluer RH, Dulaney JT, Moser HW: High performance liquid chromatography of ceramides: application to analysis in human tissues and demonstration of ceramide excess in Farber's disease. J Lipid Res 1974, 15:223-226

38. Sugita M, Connolly P, Dulaney JT, Moser HW: Fatty acid composition of free ceramides of kidney and cerebellum from a patient with Farber's disease. Lipids 1973, 8:401-406

39. Clausen J, Rampini S: Chemical studies of Farber's disease. Acta Neurol Scand 1970, 46:313-322

40. Tong M, de la Monte SM: Mechanisms of ceramide-mediated neurodegeneration. J Alzheimers Dis 2009, 16:705-714

41. Grosch S, Schiffmann S, Geisslinger G: Chain length-specific properties of ceramides. Prog Lipid Res 2012, 51:50-62

42. Cruickshanks N, Roberts JL, Bareford MD, Tavallai M, Poklepovic A, Booth L, Spiegel S, Dent P: Differential regulation of autophagy and cell viability by ceramide species. Cancer Biol Ther 2015, 16:733-742

43. Signorelli P, Munoz-Olaya JM, Gagliostro V, Casas J, Ghidoni R, Fabrias G: Dihydroceramide intracellular increase in response to resveratrol treatment mediates autophagy in gastric cancer cells. Cancer Lett 2009, 282:238-243
44. Zhao L, Spassieva SD, Jucius TJ, Shultz LD, Shick HE, Macklin WB, Hannun YA, Obeid LM, Ackerman SL: A deficiency of ceramide biosynthesis causes cerebellar Purkinje cell neurodegeneration and lipofuscin accumulation. PLoS Genet 2011, 7:e1002063

45. Hu W, Xu R, Sun W, Szulc ZM, Bielawski J, Obeid LM, Mao C: Alkaline ceramidase 3 (ACER3) hydrolyzes unsaturated long-chain ceramides, and its down-regulation inhibits both cell proliferation and apoptosis. J Biol Chem 2010, 285:7964-7976

46. Filippov V, Song MA, Zhang K, Vinters HV, Tung S, Kirsch WM, Yang J, Duerksen-Hughes PJ: Increased ceramide in brains with Alzheimer's and other neurodegenerative diseases. J Alzheimers Dis 2012, 29:537-547

47. Han X, Holtzman DM, McKeel DW Jr: Plasmalogen deficiency in early Alzheimer's disease subjects and in animal models: molecular characterization using electrospray ionization mass spectrometry. J Neurochem 2001, 77:1168-1180

48. Wang G, Dinkins M, He Q, Zhu G, Poirier C, Campbell A, Mayer-Proschel M, Bieberich E: Astrocytes secrete exosomes enriched with proapoptotic ceramide and prostate apoptosis response 4 (PAR-4): potential mechanism of apoptosis induction in Alzheimer disease (AD). J Biol Chem 2012, 287:21384-21395

49. Puglielli L, Ellis BC, Saunders AJ, Kovacs DM: Ceramide stabilizes betasite amyloid precursor protein-cleaving enzyme 1 and promotes amyloid beta-peptide biogenesis. J Biol Chem 2003, 278:19777-19783

50. Abbott SK, Li H, Munoz SS, Knoch B, Batterham M, Murphy KE, Halliday GM, Garner B: Altered ceramide acyl chain length and ceramide synthase gene expression in Parkinson's disease. Mov Disord 2014, 29:518-526

51. Vanni N, Fruscione F, Ferlazzo E, Striano P, Robbiano A, Traverso M, Sander T, Falace A, Gazzerro E, Bramanti P, Bielawski J, Fassio A, Minetti C, Genton P, Zara F: Impairment of ceramide synthesis causes a novel progressive myoclonus epilepsy. Ann Neurol 2014, 76:206-212

52. de la Monte SM, Tong M: Brain metabolic dysfunction at the core of Alzheimer's disease. Biochem Pharmacol 2014, 88:548-559

53. Chen CS, Patterson MC, Wheatley CL, O'Brien JF, Pagano RE: Broad screening test for sphingolipid-storage diseases. Lancet 1999, 354:901-905 